\title{
BMI open Validation of prescribing appropriateness criteria for older Australians using the RAND/UCLA
appropriateness method
}

\author{
Benjamin Joseph Basger, Timothy Frank Chen, Rebekah Jane Moles
}

To cite: Basger BJ, Chen TF, Moles RJ. Validation of prescribing appropriateness criteria for older Australians using the RAND/UCLA appropriateness method. BMJ Open 2012;2:e001431. doi:10.1136/bmjopen-2012001431

- Prepublication history and additional material for this paper are available online. To view these files please visit the journal online (http://dx. doi.org/10.1136/bmjopen2012-001431).

Received 17 May 2012 Accepted 20 August 2012

This final article is available for use under the terms of the Creative Commons Attribution Non-Commercial 2.0 Licence; see http://bmjopen.bmj.com

Faculty of Pharmacy, The University of Sydney, Sydney, Australia

Correspondence to Benjamin Joseph Basger; ben.basger@sydney.edu.au

\section{ABSTRACT}

Objective: To further develop and validate previously published national prescribing appropriateness criteria to assist in identifying drug-related problems (DRPs) for commonly occurring medications and medical conditions in older ( $\geq 65$ years old) Australians.

Design: RAND/UCLA appropriateness method. Participants: A panel of medication management experts were identified consisting of geriatricians/ pharmacologists, clinical pharmacists and disease management advisors to organisations that produce Australian evidence-based therapeutic publications. This resulted in a round-one panel of 15 members, and a round-two panel of 12 members.

Main outcome measure: Agreement on all criteria. Results: Forty-eight prescribing criteria were rated. In the first rating round via email, there was disagreement regarding 17 of the criteria according to median panel ratings. During a face-to-face second round meeting, discussion resulted in retention of 25 criteria after amendments, agreement for 14 criteria with no changes required and deletion of 9 criteria. Two new criteria were added, resulting in a final validated list of 41 prescribing appropriateness criteria. Agreement after round two was reached for all 41 criteria, measured by median panel ratings and the amount of dispersion of panel ratings, based on the interpercentile range.

Conclusions: A set of 41 Australian prescribing appropriateness criteria were validated by an expert panel. Use of these criteria, together with clinical judgement and other medication review processes such as patient interview, is intended to assist in improving patient care by efficiently detecting potential DRPs related to commonly occurring medicines and medical conditions in older Australians. These criteria may also contribute to the medication management education of healthcare professionals.

\section{INTRODUCTION}

Drug-related problems (DRPs) in older people ( $\geq 65$ years old) are common. ${ }^{1-4}$ They may result in drug treatment goals not being

\section{ARTICLE SUMMARY}

Article focus

- Drug-related problems (DRPs) are common in older people. They may result in drug treatment goals not being achieved and/or the occurrence of adverse drug events.

- The aim of this study was to further develop and validate a previously published list of prescribing appropriateness criteria for use in older people which may be used to improve the quality of the Australian medication review process, and for quality assessment and education in medicine use.

Key messages

- The use of medication assessment criteria is one method to assist in identifying DRPs. Criteria developed elsewhere may have little or no applicability to the Australian healthcare environment.

- Validation of proposed Australian prescribing appropriateness criteria for older people was accomplished using a two-round-modified Delphi method, resulting in agreement for all criteria as measured by median panel ratings, and the amount of dispersion of panel ratings, based on the interpercentile range.

- Use of these criteria, together with other Australian medication review processes, may assist in improving patient care in a variety of settings by efficiently identifying DRPs to common medical conditions and commonly used medicines. They may also contribute to the medication management knowledge of healthcare professionals through education programmes and by use in daily practice, and for the evaluation of the quality of pharmaceutical care in older people.

Strengths and limitations of this study

- A validated consensus method was used involving an expert medication management panel of varied specialisation. Criteria were based on established evidence-practice gaps and degree of disease burden imposed on the healthcare system, and were written with the aim of conciseness and clarity.

- Further developmental work is required to assess the usefulness of these criteria, which only included commonly occurring medicines and medical conditions. 
achieved and/or disproportionately high numbers of serious adverse medication events due to polypharmacy. ${ }^{5-7}$ DRPs can occur for many reasons such as undertreatment, inadequate monitoring of medicines, poor medicine or dose selection, duplication of medicines or factors to do with the way the patient uses the medicine. ${ }^{2}{ }^{3}{ }^{8-12}$ Methods to identify and reduce DRPs include healthcare professional-directed educational interventions, ${ }^{13}$ comprehensive geriatric assessment, ${ }^{14}$ discontinuation of multiple medications, ${ }^{15} 16$ electronic health record clinical decision support targeted towards certain diseases or drugs, ${ }^{17} 18$ and the use of medication assessment criteria, which usually consist of explicit (ie, criterion-based rather than implicit or judgement-based) lists of prescribing recommendations for various drugs and/or disease states. ${ }^{13}$ 19-22

In Australia, identification and resolution of DRPs are intended to be considered when patients are interviewed by an accredited pharmacist as part of the Home Medicines Review programme. ${ }^{23}$ This programme aims to provide the sophistication lacking in the application of explicit measures alone, as it takes into account other issues such as the patients history and personal preferences, and is targeted towards patients who may be (among other reasons) currently taking $\geq 5$ regular medicines, attending a number of different doctors, or have recently been discharged from hospital. ${ }^{24}$

In 2008, we proposed a list of 48 prescribing appropriateness criteria (45 explicit and three implicit) aimed at improving detection of DRPs as part of the Australian medication review process. $^{25}$ These criteria were intended to be applied alongside the patient interview in order to prompt appropriate history taking, particularly with respect to commonly occurring medical conditions and medicines. Similar criteria derived outside Australia have been found to have application in a variety of settings and for a variety of uses, such as in the training of healthcare professionals and in the evaluation of the quality of healthcare..$^{19}{ }^{26-29}$ Our criteria were based on the most frequent medicines prescribed to Australians, and the most frequent medical conditions for which older Australians ( $\geq 65$ years old) consult medical practitioners. Australian medication and disease state resources and guidelines were used to provide content validity. ${ }^{25}$ However, unlike our criteria, other prescribing criteria or tools have combined evidence with expert opinion to provide face validity.

The aim of this study was to further develop our list of criteria, supplementing it with recommendations for comorbidity and the oldest old where possible, and adding new criteria where necessary through expert consensus. In older patients, the importance of traditional outcomes, such as discrete clinical events or mortality, may be secondary to maintaining physical or cognitive function or relief of symptoms. ${ }^{30}$ Because of this, optimal care requires clinical decision support tools that consider issues such as patient preferences, frailty, cost and comordidities. ${ }^{31}$ Additionally, few criteria target the oldest old ${ }^{32}$ (generally regarded as people older than 85 years), where evidence may be poor, and preventive interventions may be encouraged in patients who have already exceeded an average lifespan. ${ }^{33} 34$

To further develop and validate our criteria list, we identified a panel of medication management experts, and chose the RAND/UCLA appropriateness method, which has been described as the best method for systematically combining recommendations from clinical guidelines, with the opinion of healthcare providers. ${ }^{35}$

\section{METHODS}

Ethics

Ethics approval was obtained from the Human Research Ethics Committee of the University of Sydney.

\section{Criteria development}

In 2008, we identified the 50 highest-volume Australian Pharmaceutical Benefits Scheme (PBS) medicines prescribed, and the 40 most common reasons for older Australians to seek or receive healthcare. Healthcare information was obtained using the BEACH (Bettering The Evaluation and Care of Health) programme, which continuously collects information about the clinical activities in general practice in Australia. ${ }^{36}$ We then used Australian medication information sources to identify both optimal and inappropriate medication management of these common conditions. ${ }^{25}$ In Australia, medication availability and use are largely determined by the PBS. $^{37}$ In October 2011, commonly used medications and medical conditions were checked and updated using the BEACH programme to ensure that criteria content was current. Changes in evidence, product information, Australian consensus documents, evidencebased publication recommendations or clinical practice guidelines relating to our criteria were noted for evaluation by an expert medication management panel. The criteria were designed to provide guidance on the process of care wherever it occurred-community, hospital, residential home, care home or nursing home. Major considerations in their development were likely accessibility of data from the patient, their medical notes and/or their healthcare professional(s), conciseness and clarity of wording, and provision of a practical number of criteria. Most were explicit to enable consistent application, with additional notes provided for interpretation where necessary. They were written as a statement of the kind of medication management that should or should not occur, to simplify comprehension and facilitate uptake. $^{25}$

\section{Validation of criteria: participants}

We recruited a multidisciplinary group of medication management experts to review, update and rate the criteria, consisting of geriatrician/pharmacologists, clinical pharmacists and disease management advisors to organisations that produce Australian evidence-based 
therapeutic publications. This resulted in a round-one panel of 15 members. The geriatricians consisted of two professors of geriatric medicine; an associate professor of clinical pharmacology and aged care; a research fellow in geriatric medicine and a hospital staff geriatrician. Clinical pharmacists consisted of a residential medication management review pharmacist; a home medicines review pharmacist; four hospital-based pharmacists (two team leaders, one director and one education and training pharmacist) and a professor of aged care (pharmacy). Disease management advisors to Australian evidence-based therapeutic organisations consisted of Therapeutic Guidelines, ${ }^{38}$ Australian Medicines Handbook $^{39}$ and the New South Wales Therapeutic Advisory Group. ${ }^{40}$

\section{Choice of the RAND/UCLA appropriateness method}

We chose the RAND/UCLA appropriateness method, a two-round modified Delphi method ${ }^{41}$ to select the most appropriate criteria. Unlike the Delphi method, which generally involves multiple questionnaire-driven rounds to obtain convergence of opinion, the RAND method involves an initial individual rating round, and a second face-to-face round. This method has been shown to produce results that have face, construct and predictive validity. ${ }^{42}{ }^{43}$ Systematically combining available evidence with expert opinion can create quality criteria where best evidence may be lacking. ${ }^{44}$

While most lists of prescribing criteria are based on expert consensus, this has often been achieved through mail surveys rather than face-to-face meetings. ${ }^{32} 35 \quad 45$ Although face-to-face meetings restrict panel size, they allow discussion to resolve misinterpretations, introduce new evidence and improve clarity of criteria between rating rounds. We ensured our panel comprised different specialities, as less disagreement has been found among same-specialty panels. ${ }^{46}$ We addressed concern regarding potential intimidation due to dominant panel personalities by choosing a moderator experienced in the development of these criteria and in facilitating small group discussion. This may also have assisted with conflict-of-interest issues. We used both the median panel rating and the amount of dispersion of panel ratings to identify agreement or disagreement. While it has been acknowledged that discrepancies between these two methods may occur, ${ }^{41}$ our aim was to achieve agreement for all accepted criteria for both methods after second round discussion.

\section{RAND/UCLA appropriateness method round one}

In October 2011, candidate panel members were emailed an explanation of the project and an invitation to participate. After acceptance, they were emailed a rating sheet consisting of 48 criteria, and asked to rate each on a nine-point scale. Ratings of 1-3 were classified as inappropriate, with a rating of one indicating the greatest degree of inappropriateness. Ratings of 7-9 were classified as appropriate, with a rating of nine indicating the greatest degree of appropriateness. Ratings of 4-6 were classified as neither appropriate nor inappropriate. Appropriate was defined as 'the expected health benefit exceeds the expected negative consequences by a sufficiently wide margin that criteria are worth following, exclusive of cost'. They also received a description of the way in which the criteria had been derived, and a comparison with other prescribing criteria. ${ }^{25} 32$ Panel members were requested to amend the wording or delete, update or identify missing criteria as required. Upon return of the rating sheets, results were tabulated. Agreement was based on four or less panellists rating outside the three-point region containing the median (1-3; 4-6; 7-9), and disagreement was based on five or more panellists rating in each extreme (1-3 and 7-9), as per the RAND/UCLA protocol for a 15-member panel. ${ }^{41}$

\section{Rand/UCLA appropriateness method round two}

In November 2011, a face-to-face meeting of the expert panel, chaired by a panel moderator experienced in facilitating group discussions and criteria development, met to discuss the results of round one and re-rate each of the criteria and any potential additional criteria. One pharmacist, one staff geriatrician and a disease management advisor for a therapeutics publication could not attend, resulting in a 12-member panel. For this meeting, each panel member was provided with a copy of the results from round one. This consisted of the frequency distribution of ratings of all panellists across the ninepoint scale, the overall panel median rating for each of the criteria and, for each panellist, an annotation of how they had rated each of the criteria. Scores from other panel members were not revealed. Depending on panellists votes, panel agreement or disagreement was also stated for each of the round one criteria. Additionally, the 30th and 70th percentiles adjusted for symmetry were computed for each of the criteria, as it has been found that when ratings were symmetric with respect to the middle (five on the 1-9 scale), the interpercentile range (IPR) required to label an indication as disagreement was smaller than when they were asymmetric with respect to the middle (values far from five on the 1-9 scale). Agreement after round two occurred when the IPR adjusted for symmetry (IPRAS) was greater than the IPR. ${ }^{41}$

We used the median method to present data at the face-to-face meeting, as it provided a clear visual interpretation of the ratings for each criterion. By the end of the meeting, our aim was to ensure that there was agreement between the median method and the interpercentile method for all accepted criteria.

Discussion at round two occurred on the level of agreement for each of the criteria. In addition, discussion was facilitated on the wording of each of the criteria to improve clarity and decide whether agreement would be reached. The definitions of agreement and disagreement were adjusted for the smaller second round 12 member panel. ${ }^{41}$ Agreement was reached when three or 
less panel members voted outside the three-point region containing the median, or when the IPRAS was greater than the IPR. Disagreement was determined when four or more panellists rated in each extreme (1-3 and 7-9). Each of the criteria were then discussed irrespective of whether there was agreement or disagreement, with panellists having the opportunity of changing their ratings if, for example, misinterpretation had occurred because of the way in which the criteria had been written, or if new evidence had become available, or if criteria had been interpreted in the light of a panellists own clinical experience. Each panel member consented to audio recording of the discussion. Values for the median, IPR and IPRAS ${ }^{41}$ were computed using SPSS V.20 (SPSS, Chicago, Illinois, USA).

\section{RESULTS}

After round one, there was agreement for the appropriateness of 31 of the 48 criteria, and disagreement for 17 criteria. Of the 31 criteria for which there was agreement, discussion at round two resulted in 17 criteria being amended and retained, 2 criteria being deleted and 12 criteria accepted with no change. Of the 17 criteria for which there was disagreement, discussion at round two resulted in eight criteria being amended and retained, seven criteria being deleted and two criteria accepted with no change. Two new criteria were added, resulting in a total of 41 validated criteria.

An example of how the RAND/UCLA method was applied to each of our criteria is described in table 1 for criterion one. The larger the IPRAS, the less asymmetric are the ratings. For example, 13 of 15 panellists at round one rated indicator 14 with a score of 8 or 9 , for which the IPRAS was 8.35 .

Table 2 lists the median panel ratings, the amount of dispersion of panel ratings, and whether there was agreement or disagreement for the original criteria and the validated criteria. It also lists the amendments made by the panel to the original criteria, and the reasons for these amendments. There was $100 \%$ agreement for both median panel ratings and dispersion of panel ratings for the validated criteria. Table 3 contains the final list of validated criteria, arranged according to disease states. Table 4 lists usage information judged to be necessary for certain criteria.

\section{DISCUSSION}

This study identified a panel of medication management experts to discuss and validate a set of 41 prescribing appropriateness criteria for commonly used medicines and medical conditions in older ( $\geq 65$ years) Australians. Panel discussion resulted in retention of 39 of the originally proposed 48 criteria, with 25 being reworded, and 14 accepted with no change. These criteria do not simply represent a list of medications to avoid in the elderly, but also address issues such as the need for additional therapy (eg, criteria 23 and 34, table 3), additional tests (eg, criteria 18-20, table 3), ineffective treatment (eg, criteria 22 and 37, table 3) and medication monitoring (eg, criteria 10 and 20, table 3). They were designed to contribute to the Australian quality use of medicines process. ${ }^{94}$ The information required to apply these criteria may be obtained from the patients or their carer, and patient medical notes and/or their healthcare professional. ${ }^{95}$ It may also be provided by a Home Medicines Review referral form from the patients' general practitioner. ${ }^{23}$ Owing to their currency and the nature of their development, we expect these criteria to make a significant contribution to the detection of DRPs in the Australian healthcare environment. For example, in a review of prescribing indicators for two conditions, ${ }^{36}$ which are common in older people in Australia-type 2

Table 1 An example of the application of the RAND/UCLA appropriateness method to one criterion (criterion one) from round one

\begin{tabular}{|c|c|c|c|}
\hline $\begin{array}{l}\text { Nine-point scale where } 1- \\
3=\text { inappropriate, } 4-6=\text { neither } \\
\text { appropriate nor inappropriate, } \\
7-9=\text { appropriate }\end{array}$ & $\begin{array}{l}\text { Number of } \\
\text { panellists rating } \\
\text { this criterion } \\
(\mathrm{n}=15)\end{array}$ & $\begin{array}{l}\text { Calculations, interpercentile } \\
\text { range method }{ }^{41}\end{array}$ & Interpretation \\
\hline $\begin{array}{l}1 \\
2 \\
3 \\
4 \\
5 \\
6 \\
7 \\
8 \\
9\end{array}$ & $\begin{array}{l}1 \\
1 \\
1 \\
5 \\
5 \\
2 \\
\text { Median }=7.0\end{array}$ & $\begin{array}{l}30 \text { th percentile }=7.0 \\
70 \text { th percentile }=8.0 \text {, interpercentile } \\
\text { range } \\
(\text { IPR })=70 \text { th-30th percentile })=1.0 \text {, } \\
\text { IPR central point (IPRCP })=30 \text { th } \\
+70 \text { th percentile divided by } 2=7.5 \\
\text { Asymmetry index }(\mathrm{Al})=(5-\mathrm{IPRCP}) \\
\text { (as an absolute value })=2.5 \\
\text { IPRAS }=(2.5+(\mathrm{Al} \times 1.5))=6.1 \text {, } \\
\text { where } 2.5 \text { is the IPR required } \\
\text { for disagreement when perfect } \\
\text { symmetry exists, and } 1.5 \text { is the } \\
\text { correction factor for asymmetry }\end{array}$ & $\begin{array}{l}\text { This criterion was accepted } \\
\text { according to the median method } \\
\text { because four or less panellists } \\
\text { voted outside the three-point } \\
\text { region containing the median } \\
\text { The IPRAS (6.1) was greater } \\
\text { than the IPR (1.0) indicating no } \\
\text { disagreement. The larger the } \\
\text { IPRAS, the less asymmetric the } \\
\text { ratings }\end{array}$ \\
\hline
\end{tabular}


Table 2 Changes made to original criteria according to agreement, disagreement and panel discussion

\begin{tabular}{|c|c|c|c|c|c|c|c|c|c|c|c|}
\hline \multirow{2}{*}{$\begin{array}{l}\text { Criteria } \\
\text { number }\end{array}$} & \multirow{2}{*}{$\begin{array}{l}\text { Original prescribing } \\
\text { appropriateness } \\
\text { criteria for older } \\
\text { ( } \geq 65 \text { years) } \\
\text { Australians } \\
\text { published in } 2008^{25} \\
\text { Patient taking an } \\
\text { antihypertensive is at } \\
\text { their target blood } \\
\text { pressure }\end{array}$} & \multicolumn{2}{|c|}{$\begin{array}{l}\text { Rating by } \\
\text { median } \\
\text { method }^{41} \\
\text { (median } \\
\text { value, } A \text {, } \\
\text { agreement; } D \text {, } \\
\text { disagreement), } \\
\mathrm{n}=15\end{array}$} & \multicolumn{2}{|c|}{$\begin{array}{l}\text { Rating by IPRAS }{ }^{1} \\
\text { method }^{41} \text { (IPR value, } \\
\text { IPRAS value, A, } \\
\text { agreement; } \\
\text { D=disagreement), } n=15\end{array}$} & \multirow{2}{*}{$\begin{array}{l}\text { Validated prescribing } \\
\text { appropriateness } \\
\text { criteria for older } \\
\text { ( } \geq 65 \text { years) } \\
\text { Australians as a } \\
\text { result of this study } \\
\text { Patient taking an } \\
\text { antihypertensive is at } \\
\text { the target blood } \\
\text { pressure appropriate } \\
\text { for them }\end{array}$} & \multicolumn{2}{|c|}{$\begin{array}{l}\text { Rating by } \\
\text { median } \\
\text { method }^{41} \\
\text { (median value, } \\
\text { A, agreement, } D \text {, } \\
\text { disagreement), } \\
\mathrm{n}=12\end{array}$} & \multicolumn{2}{|c|}{$\begin{array}{l}\text { Rating by IPRAS }^{1} \\
\text { method }^{41} \text { (IPR value, } \\
\text { IPRAS value, A, } \\
\text { agreement, D, } \\
\text { disagreement), } n=12 \\
\end{array}$} & \multirow[b]{2}{*}{$\begin{array}{l}\text { Amendment/reason } \\
\text { 'Appropriate for them' added. Current } \\
\text { blood pressure guidelines may not be } \\
\text { appropriate for all older patients }{ }^{47-49} \text {. } \\
\text { For example, in the oldest old }{ }^{50} \text {; in } \\
\text { palliative care; and for those who are/ } \\
\text { become hypotensive and/or fall }{ }^{51} 52\end{array}$} \\
\hline & & 7 & $A$ & $1.00,6.10$ & A & & 8 & A & $1.10,7.52$ & A & \\
\hline 2 & $\begin{array}{l}\text { Patient at high risk of } \\
\text { a cardiovascular } \\
\text { event is taking a } \\
\text { statin }\end{array}$ & 7 & $A$ & $1.00,6.10$ & A & $\begin{array}{l}\text { Patient at high risk of a } \\
\text { recurrent } \\
\text { cardiovascular event is } \\
\text { taking a statin }\end{array}$ & 8 & $A$ & $1.00,6.10$ & $A$ & $\begin{array}{l}\text { 'Recurrent' added to ensure use in } \\
\text { secondary prevention of } \\
\text { cardiovascular events rather than } \\
\text { primary prevention, where evidence } \\
\text { is less clear, especially in the oldest } \\
\text { old }^{33} 53-57\end{array}$ \\
\hline 3 & $\begin{array}{l}\text { Patient with IHD or a } \\
\text { history of } \mathrm{MI} \text { is taking } \\
\text { a } \beta \text {-blocker }\end{array}$ & 8 & A & $2.00,6.85$ & A & $\begin{array}{l}\text { Patient with } \mathrm{CHD} \text { or a } \\
\text { history of } \mathrm{Ml} \text { is taking a } \\
\beta \text {-blocker }\end{array}$ & 7 & $A$ & $1.00,6.10$ & A & $\begin{array}{l}\text { 'CHD' replaced 'IHD'. The term } \\
\text { 'coronary heart disease' is preferred } \\
\text { over 'ischaemic heart disease' }\end{array}$ \\
\hline 4 & $\begin{array}{l}\text { Patient with IHD or a } \\
\text { history of MI is taking } \\
\text { an antiplatelet agent } \\
\text { unless on an oral } \\
\text { anticoagulant }\end{array}$ & 8 & A & $1.00,7.60$ & A & $\begin{array}{l}\text { Patient with CHD or a } \\
\text { history of } \mathrm{Ml} \text { is taking } \\
\text { an antiplatelet agent } \\
\text { unless on an oral } \\
\text { anticoagulant }\end{array}$ & 8 & $A$ & $1.00,7.60$ & A & $\begin{array}{l}\text { 'CHD' replaced 'IHD'. The term } \\
\text { 'coronary heart disease' is preferred } \\
\text { over 'ischaemic heart disease' }\end{array}$ \\
\hline 5 & $\begin{array}{l}\text { Patient with heart } \\
\text { failure is taking a } \\
\beta \text {-blocker }\end{array}$ & 7 & A & $1.00,6.10$ & A & $\begin{array}{l}\text { Patient with stable } \\
\text { HF-LVSD is taking a } \\
\beta \text {-blocker }\end{array}$ & 8 & A & $0.10,6.78$ & A & $\begin{array}{l}\text { Description of heart failure amended. } \\
\text { The use of } \beta \text {-blockers is } \\
\text { contraindicated in unstable heart } \\
\text { failure. The optimal treatment of } \\
\text { HFPEF is uncertain at this time } \\
5859\end{array}$ \\
\hline 6 & $\begin{array}{l}\text { Patient with heart } \\
\text { failure is taking an } \\
\mathrm{ACEI} \text { or } \mathrm{A} 2 \mathrm{~A}\end{array}$ & 8 & A & $2.00,6.85$ & A & $\begin{array}{l}\text { Patient with stable } \\
\text { HF-LVSD is taking an } \\
\text { ACEI or A2A }\end{array}$ & 9 & A & $1.00,7.60$ & A & $\begin{array}{l}\text { Description of heart failure amended. } \\
\text { The optimal treatment of HFPEF is } \\
\text { uncertain at this time }{ }^{5859}\end{array}$ \\
\hline 7 & $\begin{array}{l}\text { Patient with heart } \\
\text { failure is NOT taking } \\
\text { medications which } \\
\text { may exacerbate heart } \\
\text { failure }\end{array}$ & 9 & A & $1.00,7.60$ & A & $\begin{array}{l}\text { Patient with HF-LVSD } \\
\text { or HFPEF is NOT } \\
\text { taking medications } \\
\text { which may exacerbate } \\
\text { heart failure }\end{array}$ & 9 & $A$ & $0.10,8.27$ & A & $\begin{array}{l}\text { Description of heart failure amended. } \\
\text { The types of medicines } \\
\text { contraindicated in HF-LVSD and } \\
\text { HFPEF may not be identical }\end{array}$ \\
\hline
\end{tabular}




\begin{tabular}{|c|c|c|c|c|c|c|c|c|c|c|c|}
\hline \multirow{2}{*}{$\begin{array}{l}\text { Criteria } \\
\text { number }\end{array}$} & \multirow{2}{*}{$\begin{array}{l}\text { Original prescribing } \\
\text { appropriateness } \\
\text { criteria for older } \\
\text { ( } \geq 65 \text { years) } \\
\text { Australians } \\
\text { published in } 2008^{25} \\
\text { Patient with heart } \\
\text { failure or } \\
\text { hypertension is NOT } \\
\text { taking high sodium } \\
\text { medications }\end{array}$} & \multicolumn{2}{|c|}{$\begin{array}{l}\text { Rating by } \\
\text { median } \\
\text { method }^{41} \\
\text { (median } \\
\text { value, A, } \\
\text { agreement; } D \text {, } \\
\text { disagreement), } \\
n=15\end{array}$} & \multicolumn{2}{|c|}{$\begin{array}{l}\text { Rating by IPRAS }{ }^{1} \\
\text { method }^{41} \text { (IPR value, } \\
\text { IPRAS value, } A \text {, } \\
\text { agreement; } \\
\text { D=disagreement), } n=15\end{array}$} & \multirow{2}{*}{$\begin{array}{l}\text { Validated prescribing } \\
\text { appropriateness } \\
\text { criteria for older } \\
\text { ( } \geq 65 \text { years) } \\
\text { Australians as a } \\
\text { result of this study } \\
\text { Deleted }\end{array}$} & \multicolumn{2}{|c|}{$\begin{array}{l}\text { Rating by } \\
\text { median } \\
\text { method }^{41} \\
\text { (median value, } \\
\text { A, agreement, D, } \\
\text { disagreement), } \\
n=12\end{array}$} & \multicolumn{2}{|c|}{$\begin{array}{l}\text { Rating by IPRAS }{ }^{1} \\
\text { method }^{41} \text { (IPR value, } \\
\text { IPRAS value, A, } \\
\text { agreement, } D, \\
\text { disagreement), } n=12\end{array}$} & \multirow{2}{*}{$\begin{array}{l}\text { Amendment/reason } \\
\text { High sodium medicines (among } \\
\text { others) in heart failure are addressed } \\
\text { by indicator } 7 \text {. In hypertension, they } \\
\text { are addressed as lifestyle } \\
\text { modifications } 6263\end{array}$} \\
\hline & & 8 & D & $2.20,5.50$ & A & & - & & - & - & \\
\hline 9 & $\begin{array}{l}\text { Patient with } \mathrm{AF} \text { is } \\
\text { taking an oral } \\
\text { anticoagulant }\end{array}$ & 7 & $D$ & $2.0,5.35$ & $A$ & $\begin{array}{l}\text { Patient with } \mathrm{AF} \text { is } \\
\text { taking an oral } \\
\text { anticoagulant or an } \\
\text { antiplatelet agent, } \\
\text { depending upon stroke } \\
\text { risk and bleeding risk }\end{array}$ & 8 & $A$ & $0.10,6.93$ & $A$ & $\begin{array}{l}\text { An antiplatelet agent may be } \\
\text { appropriate for patients at low risk of } \\
\text { stroke. Bleeding risk may determine } \\
\text { choice of antithrombotic agent }{ }^{49} 6465\end{array}$ \\
\hline 10 & $\begin{array}{l}\text { Patient with } \mathrm{AF} \text { taking } \\
\text { an anticoagulant has } \\
\text { an INR between } 2 \\
\text { and } 3\end{array}$ & 8 & A & $2.20,6.70$ & A & $\begin{array}{l}\text { Patient taking warfarin } \\
\text { for AF has an INR } \\
\text { between } 2 \text { and } 3\end{array}$ & 9 & $A$ & $1.00,7.60$ & $A$ & $\begin{array}{l}\text { New anticoagulants like rivaroxaban } \\
\text { and dabigatran do not require INR } \\
\text { monitoring }\end{array}$ \\
\hline 11 & $\begin{array}{l}\text { Patient with a history } \\
\text { of non-haemorrhagic } \\
\text { stroke or TIA is taking } \\
\text { an antiplatelet agent } \\
\text { unless on an } \\
\text { anticoagulant }\end{array}$ & 8 & $A$ & $1.00,7.60$ & A & $\begin{array}{l}\text { Patient with a history of } \\
\text { non-haemorrhagic } \\
\text { stroke or TIA is taking } \\
\text { an antiplatelet agent } \\
\text { unless on an } \\
\text { anticoagulant }\end{array}$ & 9 & A & $1.00,7.60$ & A & No change \\
\hline 12 & $\begin{array}{l}\text { Patient with risk } \\
\text { factors for myopathy } \\
\text { is NOT taking } 40 \mathrm{mg} \\
\text { or more per day of } \\
\text { simvastatin or } \\
\text { atorvastatin }\end{array}$ & 7 & D & $3.00,4.60$ & A & $\begin{array}{l}\text { Patient with risk factors } \\
\text { for statin-induced } \\
\text { myopathy is not taking } \\
\text { a high dose of a } \\
\text { high-potency statin }\end{array}$ & 8 & A & $1.10,7.52$ & $A$ & $\begin{array}{l}\text { The use of all high dose of } \\
\text { high-potency statins together with } \\
\text { risk factors may increase the } \\
\text { likelihood of myopathy } 49667\end{array}$ \\
\hline 13 & $\begin{array}{l}\text { Patient with } \\
\text { cardiovascular } \\
\text { disease is NOT taking } \\
\text { an NSAID }\end{array}$ & 7 & A & $1.20,5.95$ & A & $\begin{array}{l}\text { Patient with } \\
\text { cardiovascular disease } \\
\text { is NOT taking an } \\
\text { NSAID }\end{array}$ & 8 & A & $1.10,6.18$ & $A$ & No change \\
\hline
\end{tabular}




\begin{tabular}{|c|c|c|c|c|c|c|c|c|c|c|c|}
\hline \multirow{2}{*}{$\begin{array}{l}\text { Criteria } \\
\text { number }\end{array}$} & \multirow{2}{*}{$\begin{array}{l}\text { Original prescribing } \\
\text { appropriateness } \\
\text { criteria for older } \\
\text { ( } \geq 65 \text { years) } \\
\text { Australians } \\
\text { published in } 200 \mathbf{8}^{25} \\
\text { Patient with } \\
\text { cardiovascular, } \\
\text { respiratory disease or } \\
\text { diabetes who smokes } \\
\text { has been offered } \\
\text { smoking cessation } \\
\text { therapy }\end{array}$} & \multicolumn{2}{|c|}{$\begin{array}{l}\text { Rating by } \\
\text { median } \\
\text { method }^{41} \\
\text { (median } \\
\text { value, A, } \\
\text { agreement; } D \text {, } \\
\text { disagreement), } \\
n=15\end{array}$} & \multicolumn{2}{|c|}{$\begin{array}{l}\text { Rating by IPRAS }{ }^{1} \\
\text { method }^{41} \text { (IPR value, } \\
\text { IPRAS value, A, } \\
\text { agreement; } \\
\text { D=disagreement), } n=15\end{array}$} & \multirow{2}{*}{$\begin{array}{l}\text { Validated prescribing } \\
\text { appropriateness } \\
\text { criteria for older } \\
\text { ( } \geq 65 \text { years) } \\
\text { Australians as a } \\
\text { result of this study } \\
\text { Patient with } \\
\text { cardiovascular, } \\
\text { respiratory disease or } \\
\text { diabetes who smokes } \\
\text { has been offered } \\
\text { smoking cessation } \\
\text { options }\end{array}$} & \multicolumn{2}{|c|}{$\begin{array}{l}\text { Rating by } \\
\text { median } \\
\text { method }^{41} \\
\text { (median value, } \\
\text { A, agreement, } D \text {, } \\
\text { disagreement), } \\
\mathrm{n}=12\end{array}$} & \multicolumn{2}{|c|}{$\begin{array}{l}\text { Rating by IPRAS }{ }^{1} \\
\text { method }^{41} \text { (IPR value, } \\
\text { IPRAS value, } A \text {, } \\
\text { agreement, } D, \\
\text { disagreement), } n=12\end{array}$} & \multirow{2}{*}{$\begin{array}{l}\text { Amendment/reason } \\
\text { 'Therapy' implies pharmacotherapy, } \\
\text { whereas repeated counselling/ } \\
\text { psychotherapy may be preferred to } \\
\text { avoid the risks associated with } \\
\text { polypharmacy }\end{array}$} \\
\hline & & 9 & A & $0.00,8.35$ & $A$ & & 9 & $A$ & $0.00,8.35$ & $A$ & \\
\hline 15 & $\begin{array}{l}\text { Patient with type } 2 \\
\text { diabetes and } \\
\text { hypertension and } \\
\text { albuminuria is taking } \\
\text { an ACEI or A2A }\end{array}$ & 8 & A & $2.00,6.85$ & A & $\begin{array}{l}\text { Patient with type } 2 \\
\text { diabetes and } \\
\text { hypertension and } \\
\text { albuminuria is taking an } \\
\text { ACEI or A2A }\end{array}$ & 9 & A & $1.00,7.60$ & A & No change \\
\hline 16 & $\begin{array}{l}\text { Patient with diabetes } \\
\text { at high risk of a } \\
\text { cardiovascular event } \\
\text { is taking an } \\
\text { antiplatelet agent } \\
\text { unless on an } \\
\text { anticoagulant }\end{array}$ & 7 & D & $2.20,5.50$ & A & $\begin{array}{l}\text { Patient with diabetes at } \\
\text { high risk of a } \\
\text { cardiovascular event is } \\
\text { taking an antiplatelet } \\
\text { agent unless on an } \\
\text { anticoagulant }\end{array}$ & 9 & $A$ & $1.00,7.60$ & A & No change \\
\hline 17 & $\begin{array}{l}\text { Patient with diabetes } \\
\text { is NOT taking a } \\
\text { medication which may } \\
\text { increase or decrease } \\
\text { blood glucose } \\
\text { concentrations }\end{array}$ & 5 & D & $2.20,3.70$ & $A$ & $\begin{array}{l}\text { Patient with diabetes } \\
\text { receiving medications } \\
\text { that may affect } \\
\text { glycaemic control is } \\
\text { having regular } \\
\text { monitoring of blood } \\
\text { glucose concentrations }\end{array}$ & 9 & $A$ & $1.00,7.60$ & A & $\begin{array}{l}\text { Increased awareness and monitoring } \\
\text { may require adjustment of } \\
\text { hypoglycaemic medication doses, } \\
\text { depending on the need to continue } \\
\text { interacting medicines. For example, } \\
\text { the start of oral corticosteroids may } \\
\text { worsen diabetes control }^{39}\end{array}$ \\
\hline 18 & $\begin{array}{l}\text { Patient with diabetes } \\
\text { has had an } \mathrm{HbA1c} \\
\text { measurement within } \\
\text { the previous } 6 \text { months }\end{array}$ & 8 & $A$ & $1.20,7.45$ & A & $\begin{array}{l}\text { Patient with diabetes } \\
\text { has had an } \mathrm{HbA} 1 \mathrm{c} \\
\text { measurement within } \\
\text { the previous } 6 \text { months }\end{array}$ & 8 & $A$ & $1.00,7.60$ & A & No change \\
\hline 19 & $\begin{array}{l}\text { Patient taking } \\
\text { metformin for } \\
\text { diabetes has had the } \\
\text { dose adjusted for } \\
\text { creatinine clearance }\end{array}$ & 8 & $A$ & $1.20,7.45$ & A & $\begin{array}{l}\text { Patient taking } \\
\text { metformin for diabetes } \\
\text { has had the dose } \\
\text { adjusted for renal } \\
\text { function }\end{array}$ & 9 & $A$ & $1.00,7.60$ & $A$ & $\begin{array}{l}\text { Creatinine clearance may represent } \\
\text { only one of the methods used to } \\
\text { determine renal function }\end{array}$ \\
\hline
\end{tabular}




\begin{tabular}{|c|c|c|c|c|c|c|c|c|c|}
\hline \multirow{2}{*}{$\begin{array}{l}\text { Criteria } \\
\text { number }\end{array}$} & \multirow{2}{*}{$\begin{array}{l}\text { Original prescribing } \\
\text { appropriateness } \\
\text { criteria for older } \\
\text { ( } \geq 65 \text { years) } \\
\text { Australians } \\
\text { published in } 2008^{25} \\
\text { Patient taking } \\
\text { metformin for } \\
\text { diabetes is NOT } \\
\text { concurrently taking } \\
\text { glibenclamide }\end{array}$} & $\begin{array}{l}\text { Rating by } \\
\text { median } \\
\text { method }^{41} \\
\text { (median } \\
\text { value, } A \text {, } \\
\text { agreement; } D \text {, } \\
\text { disagreement), } \\
n=15\end{array}$ & \multicolumn{2}{|c|}{$\begin{array}{l}\text { Rating by IPRAS }{ }^{1} \\
\text { method }^{41} \text { (IPR value, } \\
\text { IPRAS value, A, } \\
\text { agreement; } \\
\text { D=disagreement), } n=15\end{array}$} & \multirow{2}{*}{$\begin{array}{l}\text { Validated prescribing } \\
\text { appropriateness } \\
\text { criteria for older } \\
\text { ( } \geq 65 \text { years) } \\
\text { Australians as a } \\
\text { result of this study } \\
\text { Deleted }\end{array}$} & $\begin{array}{l}\text { Rating by } \\
\text { median } \\
\text { method }^{41} \\
\text { (median value, } \\
\text { A, agreement, D, } \\
\text { disagreement), } \\
n=12\end{array}$ & \multicolumn{2}{|c|}{$\begin{array}{l}\text { Rating by IPRAS }{ }^{1} \\
\text { method }^{41} \text { (IPR value, } \\
\text { IPRAS value, } A \text {, } \\
\text { agreement, } D \text {, } \\
\text { disagreement), } n=12\end{array}$} & \multirow{2}{*}{$\begin{array}{l}\text { Amendment/reason } \\
\text { Glibenclamide is an uncommonly } \\
\text { used hypoglycaemic }\end{array}$} \\
\hline & & $\mathrm{D}$ & $2.40,3.85$ & A & & - & - & - & \\
\hline 21 & $\begin{array}{l}\text { Patient with OA pain } \\
\text { interfering with daily } \\
\text { activities has been } \\
\text { trialled on } \\
\text { paracetamol 2-4 } \\
\text { g/day }\end{array}$ & $A$ & $2.00,6.85$ & A & $\begin{array}{l}\text { Patient with OA pain } \\
\text { interfering with daily } \\
\text { activities has been } \\
\text { trialled on regular } \\
\text { paracetamol } 2-4 \mathrm{~g} / \text { day }\end{array}$ & 9 & $0.40,8.05$ & A & $\begin{array}{l}\text { 'Regular' paracetamol added to } \\
\text { improve quality of indicator }\end{array}$ \\
\hline 22 & $\begin{array}{l}\text { Patient taking } \\
\text { analgesic(s) does } \\
\text { NOT have pain that } \\
\text { interferes with daily } \\
\text { activities }\end{array}$ & $\mathrm{D}$ & $3.2,4.75$ & A & $\begin{array}{l}\text { Patient taking analgesic } \\
\text { (s) has had the dose(s) } \\
\text { titrated in order to avoid } \\
\text { pain that interferes with } \\
\text { daily activities }\end{array}$ & 8 & $2.00,6.85$ & A & Indicator rephrased to improve clarity \\
\hline 23 & $\begin{array}{l}\text { Patient taking an } \\
\text { opioid is on } \\
\text { prophylactic treatment } \\
\text { for constipation }\end{array}$ & A & $2.00,6.85$ & A & $\begin{array}{l}\text { Patient taking a regular } \\
\text { opioid is on } \\
\text { prophylactic treatment } \\
\text { for constipation }\end{array}$ & 9 & $1.00,7.60$ & $A$ & $\begin{array}{l}\text { 'Regular' use added as 'when } \\
\text { required' use may not always require } \\
\text { prophylactic treatment }\end{array}$ \\
\hline 24 & $\begin{array}{l}\text { Patient with risk } \\
\text { factors for impaired } \\
\text { renal function is NOT } \\
\text { taking an NSAID }\end{array}$ & A & $1.00,7.60$ & A & $\begin{array}{l}\text { Patient with risk factors } \\
\text { for impaired renal } \\
\text { function is NOT taking } \\
\text { an NSAID }\end{array}$ & 8 & $1.00,7.60$ & A & No change \\
\hline 25 & $\begin{array}{l}\text { Patient is NOT } \\
\text { concurrently taking } \\
\text { an ACEI or A2A, } \\
\text { diuretic and NSAID } \\
\text { (excluding low-dose } \\
\text { aspirin) }\end{array}$ & A & $1.00,7.60$ & A & $\begin{array}{l}\text { Patient is NOT } \\
\text { concurrently taking an } \\
\text { ACEI or A2A, diuretic } \\
\text { and NSAID (excluding } \\
\text { low-dose aspirin) }\end{array}$ & 9 & $1.00,7.60$ & A & No change \\
\hline 26 & $\begin{array}{l}\text { Patient with sleep } \\
\text { disturbance or anxiety } \\
\text { has NOT been taking } \\
\text { benzodiazepines for } \\
\text { >4 weeks }\end{array}$ & 8 & $1.20,7.45$ & A & $\begin{array}{l}\text { Patient has NOT been } \\
\text { taking benzodiazepines } \\
\text { for }>4 \text { weeks }\end{array}$ & 9 & $1.00,7.60$ & A & $\begin{array}{l}\text { 'Sleep disturbance or anxiety' } \\
\text { deleted. Benzodiazepines increase } \\
\text { the risk of oversedation, ataxia, } \\
\text { confusion, falls, respiratory } \\
\text { depression and short-term memory } \\
\text { impairment, and are recommended } \\
\text { for short-term use only }\end{array}$ \\
\hline
\end{tabular}




\begin{tabular}{|c|c|c|c|c|c|c|c|c|c|c|c|}
\hline \multirow{2}{*}{$\begin{array}{l}\begin{array}{l}\text { Criteria } \\
\text { number }\end{array} \\
27\end{array}$} & \multirow{2}{*}{$\begin{array}{l}\text { Original prescribing } \\
\text { appropriateness } \\
\text { criteria for older } \\
\text { ( } \geq 65 \text { years) } \\
\text { Australians } \\
\text { published in } 200 \mathbf{8}^{25} \\
\text { Patient with } \\
\text { depression is NOT } \\
\text { taking } \\
\text { anticholinergic-type } \\
\text { antidepressants }\end{array}$} & \multicolumn{2}{|c|}{$\begin{array}{l}\text { Rating by } \\
\text { median } \\
\text { method }^{41} \\
\text { (median } \\
\text { value, } A \text {, } \\
\text { agreement; } D \text {, } \\
\text { disagreement), } \\
\mathrm{n}=15\end{array}$} & \multicolumn{2}{|c|}{$\begin{array}{l}\text { Rating by IPRAS }{ }^{1} \\
\text { method }^{41} \text { (IPR value, } \\
\text { IPRAS value, A, } \\
\text { agreement; } \\
\text { D=disagreement), } n=15\end{array}$} & \multirow[t]{2}{*}{$\begin{array}{l}\text { Validated prescribing } \\
\text { appropriateness } \\
\text { criteria for older } \\
\text { ( } \geq 65 \text { years) } \\
\text { Australians as a } \\
\text { result of this study } \\
\text { Deleted }\end{array}$} & \multicolumn{2}{|c|}{$\begin{array}{l}\text { Rating by } \\
\text { median } \\
\text { method }^{41} \\
\text { (median value, } \\
A \text {, agreement, } D \text {, } \\
\text { disagreement), } \\
n=12\end{array}$} & \multicolumn{2}{|c|}{$\begin{array}{l}\text { Rating by IPRAS }{ }^{1} \\
\text { method }^{41} \text { (IPR value, } \\
\text { IPRAS value, } A \text {, } \\
\text { agreement, } D, \\
\text { disagreement), } n=12\end{array}$} & \multirow{2}{*}{$\begin{array}{l}\text { Amendment/reason } \\
\text { The issue of anticholinergic burden is } \\
\text { addressed by indicator } 32\end{array}$} \\
\hline & & 7 & D & $1.00,4.60$ & $A$ & & - & & - & - & \\
\hline 28 & $\begin{array}{l}\text { Patient with a history } \\
\text { of falls is NOT taking } \\
\text { psychotropic } \\
\text { medications }\end{array}$ & 8 & $A$ & $1.00,6.10$ & A & $\begin{array}{l}\text { Patient with a history of } \\
\text { falls is NOT taking } \\
\text { psychotropic } \\
\text { medications }\end{array}$ & 8 & A & $1.40,6.40$ & A & No change \\
\hline 29 & $\begin{array}{l}\text { Patient taking an } \\
\text { SSRI is NOT } \\
\text { concurrently taking } \\
\text { medications known to } \\
\text { increase the risk of GI } \\
\text { bleeding }\end{array}$ & 7 & D & $2.20,5.20$ & $A$ & Deleted & - & & - & - & $\begin{array}{l}\text { Redundant indicator. This issue } \\
\text { would be identified by indicator } 47\end{array}$ \\
\hline 30 & $\begin{array}{l}\text { Patient taking an } \\
\text { SSRI is NOT } \\
\text { concurrently taking } \\
\text { other medications that } \\
\text { may contribute to } \\
\text { serotonin toxicity }\end{array}$ & 8 & $A$ & $2.20,6.70$ & A & $\begin{array}{l}\text { Patient taking an SSRI } \\
\text { is NOT concurrently } \\
\text { taking other } \\
\text { medications that may } \\
\text { contribute to serotonin } \\
\text { toxicity }\end{array}$ & 8 & $A$ & $1.40,6.40$ & $A$ & $\begin{array}{l}\text { No change. Retained by panel due to } \\
\text { its potential significance, despite the } \\
\text { use of indicator } 47\end{array}$ \\
\hline 31 & $\begin{array}{l}\text { Patient with dementia } \\
\text { is NOT receiving } \\
\text { anticholinergic } \\
\text { medication }\end{array}$ & 8 & $A$ & $1.20,7.45$ & A & $\begin{array}{l}\text { Patient with dementia is } \\
\text { NOT receiving } \\
\text { anticholinergic } \\
\text { medication }\end{array}$ & 8 & A & $1.00,7.60$ & $A$ & No change \\
\hline 32 & $\begin{array}{l}\text { Patient is NOT taking } \\
\text { more than one } \\
\text { medication with } \\
\text { anticholinergic activity }\end{array}$ & 8 & A & $0.2,6.70$ & A & $\begin{array}{l}\text { Patient is not taking } \\
\text { medication with } \\
\text { SIGNIFICANT } \\
\text { anticholinergic activity }\end{array}$ & 8 & A & $0.40,7.15$ & A & $\begin{array}{l}\text { Rewording focuses on the issue of } \\
\text { anticholinergic burden }\end{array}$ \\
\hline 33 & $\begin{array}{l}\text { Patient taking a PPI is } \\
\text { NOT taking a } \\
\text { medication that may } \\
\text { cause dyspepsia }\end{array}$ & 7 & $D$ & $3.20,4.45$ & A & $\begin{array}{l}\text { Patient taking a PPI is } \\
\text { NOT taking a } \\
\text { medication that may } \\
\text { cause dyspepsia } \\
\text { unless prescribed for } \\
\text { gastroprotection }\end{array}$ & 8 & A & $0.40,7.15$ & A & $\begin{array}{l}\text { 'Unless prescribed for } \\
\text { gastroprotection' added to improve } \\
\text { the accuracy of the indicator }\end{array}$ \\
\hline
\end{tabular}




\begin{tabular}{|c|c|c|c|c|c|c|c|c|c|c|c|}
\hline \multirow{2}{*}{$\begin{array}{l}\text { Criteria } \\
\text { number }\end{array}$} & \multirow{2}{*}{ 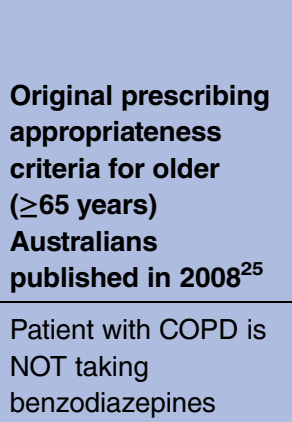 } & \multicolumn{2}{|c|}{$\begin{array}{l}\text { Rating by } \\
\text { median } \\
\text { method }^{41} \\
\text { (median } \\
\text { value, } A \text {, } \\
\text { agreement; } D \text {, } \\
\text { disagreement), } \\
\mathrm{n}=15\end{array}$} & \multicolumn{2}{|c|}{$\begin{array}{l}\text { Rating by IPRAS }{ }^{1} \\
\text { method }^{4} \text { (IPR value, } \\
\text { IPRAS value, A, }^{\text {agreement; }} \\
\text { D=disagreement), } n=15\end{array}$} & \multirow{2}{*}{$\begin{array}{l}\text { Validated prescribing } \\
\text { appropriateness } \\
\text { criteria for older } \\
\text { ( } \geq 65 \text { years) } \\
\text { Australians as a } \\
\text { result of this study } \\
\text { Patient with COPD is } \\
\text { NOT taking } \\
\text { benzodiazepines }\end{array}$} & \multicolumn{2}{|c|}{$\begin{array}{l}\text { Rating by } \\
\text { median } \\
\text { method }^{41} \\
\text { (median value, } \\
\text { A, agreement, } \mathrm{D} \text {, } \\
\text { disagreement), } \\
\mathrm{n}=12\end{array}$} & \multicolumn{2}{|c|}{$\begin{array}{l}\text { Rating by IPRAS }{ }^{1} \\
\text { method }^{41} \text { (IPR value, } \\
\text { IPRAS value, } A,_{\text {agreement, } D,} \\
\text { disagreement), } \mathrm{n}=12 \\
\end{array}$} & \multirow{2}{*}{$\begin{array}{l}\text { Amendment/reason } \\
\text { No change }\end{array}$} \\
\hline & & 7 & D & $3.00,6.10$ & A & & 8 & A & $1.00,6.10$ & A & \\
\hline 35 & $\begin{array}{l}\text { Patient with asthma } \\
\text { using an inhaled } \\
\text { LABA is also using an } \\
\text { inhaled corticosteroid }\end{array}$ & 9 & A & $0.20,8.20$ & A & $\begin{array}{l}\text { Patient with asthma } \\
\text { using an inhaled LABA } \\
\text { is also using an inhaled } \\
\text { corticosteroid }\end{array}$ & 9 & A & $1.00,7.60$ & A & No change \\
\hline 36 & $\begin{array}{l}\text { Patient using } \\
\text { salbutamol or } \\
\text { terbutaline inhaler } \\
\text { more than three times } \\
\text { per week for } \\
\text { reversible airways } \\
\text { disease has been } \\
\text { prescribed an ICS }\end{array}$ & 9 & A & $1.00,7.60$ & A & $\begin{array}{l}\text { Patient using } \\
\text { salbutamol or } \\
\text { terbutaline inhaler more } \\
\text { than three times per } \\
\text { week for reversible } \\
\text { airways disease has } \\
\text { been prescribed an } \\
\text { ICS (except for } \\
\text { exercise-induced } \\
\text { asthma) }\end{array}$ & 9 & A & $0.40,8.05$ & A & $\begin{array}{l}\text { 'Except for exercise-induced asthma' } \\
\text { added to improve the accuracy of the } \\
\text { indicator }\end{array}$ \\
\hline 37 & $\begin{array}{l}\text { Patient with asthma is } \\
\text { NOT taking a } \\
\text { medication that may } \\
\text { worsen asthma }\end{array}$ & 7 & A & $1.20,6.25$ & A & $\begin{array}{l}\text { Patient with asthma is } \\
\text { NOT taking a } \\
\text { medication that may } \\
\text { worsen asthma }\end{array}$ & 8 & A & $1.00,7.60$ & A & No change \\
\hline 38 & $\begin{array}{l}\text { Female patient with } \\
\text { recurrent UTIs has } \\
\text { been prescribed } \\
\text { intravaginal oestrogen }\end{array}$ & 5 & D & $2.00,3.85$ & A & Deleted & - & & - & - & $\begin{array}{l}\text { Evidence for this indicator was } \\
\text { judged to be poor }{ }^{68}\end{array}$ \\
\hline 39 & $\begin{array}{l}\text { Patient with a } \\
\text { creatinine clearance } \\
<60 \mathrm{ml} / \mathrm{min} \text { is NOT } \\
\text { receiving } \\
\text { nitrofurantoin for UTI }\end{array}$ & 8 & A & $2.00,6.85$ & A & $\begin{array}{l}\text { Patient with a UTI is } \\
\text { not receiving } \\
\text { nitrofurantoin or } \\
\text { hexamine for } \\
\text { prophylaxis or acute } \\
\text { treatment }\end{array}$ & 8 & A & $1.00,7.60$ & A & $\begin{array}{l}\text { Hexamine and nitrofurantoin are not } \\
\text { recommended for the prophylactic or } \\
\text { acute treatment of UTI in older } \\
\text { patients }^{39} 49\end{array}$ \\
\hline 40 & $\begin{array}{l}\text { Patient with a } \\
\text { creatinine clearance } \\
<50 \mathrm{ml} / \mathrm{min} \text { is NOT } \\
\text { receiving hexamine } \\
\text { for UTI prophylaxis }\end{array}$ & 8 & A & $1.20,6.25$ & A & Deleted & - & - & - & - & $\begin{array}{l}\text { Hexamine and nitrofurantoin are not } \\
\text { recommended for the prophylactic } \\
\text { treatment of UTI in older patients }{ }^{39} 49\end{array}$ \\
\hline
\end{tabular}




\begin{tabular}{|c|c|c|c|c|c|c|c|c|c|c|}
\hline \multirow{2}{*}{$\begin{array}{l}\begin{array}{l}\text { Criteria } \\
\text { number }\end{array} \\
41\end{array}$} & \multirow{2}{*}{$\begin{array}{l}\text { Original prescribing } \\
\text { appropriateness } \\
\text { criteria for older } \\
\text { ( } \geq 65 \text { years) } \\
\text { Australians } \\
\text { published in } 2008^{25} \\
\text { Patient with an URTI } \\
\text { is NOT receiving } \\
\text { antibiotics }\end{array}$} & $\begin{array}{l}\text { Rating by } \\
\text { median } \\
\text { method }^{41} \\
\text { (median } \\
\text { value, } A \text {, } \\
\text { agreement; } D \text {, } \\
\text { disagreement), } \\
\mathrm{n}=15\end{array}$ & \multicolumn{2}{|c|}{$\begin{array}{l}\text { Rating by IPRAS }{ }^{1} \\
\text { method }^{41} \text { (IPR value, } \\
\text { IPRAS value, A, } \\
\text { agreement; } \\
\text { D=disagreement), } n=15\end{array}$} & \multirow{2}{*}{$\begin{array}{l}\text { Validated prescribing } \\
\text { appropriateness } \\
\text { criteria for older } \\
\text { ( } \geq 65 \text { years) } \\
\text { Australians as a } \\
\text { result of this study } \\
\text { Patient with a } \\
\text { non-specific URTI is } \\
\text { NOT receiving } \\
\text { antibiotics }\end{array}$} & \multicolumn{2}{|c|}{$\begin{array}{l}\text { Rating by } \\
\text { median } \\
\text { method }^{41} \\
\text { (median value, } \\
\text { A, agreement, } D \text {, } \\
\text { disagreement), } \\
\mathrm{n}=12\end{array}$} & \multicolumn{2}{|c|}{$\begin{array}{l}\text { Rating by IPRAS }{ }^{1} \\
\text { method }^{41} \text { (IPR value, } \\
\text { IPRAS value, } A, \\
\text { agreement, } D, \\
\text { disagreement), } n=12\end{array}$} & \multirow{2}{*}{$\begin{array}{l}\text { Amendment/reason } \\
\text { 'Non-specific' added to improve the } \\
\text { accuracy of the indicator }\end{array}$} \\
\hline & & $\mathrm{D}$ & $3.00,4.60$ & $A$ & & 8 & A & $1.00,7.60$ & $A$ & \\
\hline 42 & $\begin{array}{l}\text { Patient with } \\
\text { osteoporosis who is } \\
\text { not receiving at least } \\
600 \text { IU vitamin D daily } \\
\text { from dietary sources } \\
\text { is receiving } \\
\text { supplementation with } \\
\text { vitamin D }\end{array}$ & D & $3.20,4.75$ & $A$ & Deleted & - & & - & - & $\begin{array}{l}\text { This indicator is covered by indicator } \\
44 \text { and an expanded footnote }\end{array}$ \\
\hline 43 & $\begin{array}{l}\text { Patient with } \\
\text { osteoporosis who is } \\
\text { not receiving at least } \\
1200 \mathrm{mg} \text { of calcium } \\
\text { daily from dietary } \\
\text { sources is receiving } \\
\text { calcium } \\
\text { supplementation }\end{array}$ & A & $1.60,5.95$ & $A$ & Deleted & - & & - & - & $\begin{array}{l}\text { This indicator is covered by indicator } \\
44 \text { and an expanded footnote }\end{array}$ \\
\hline 44 & $\begin{array}{l}\text { Patient with } \\
\text { osteoporosis is } \\
\text { receiving } \\
\text { antiosteoporotic } \\
\text { medication }\end{array}$ & $A$ & $1.00,6.10$ & $A$ & $\begin{array}{l}\text { Patient with } \\
\text { osteoporosis is } \\
\text { receiving appropriate } \\
\text { antiosteoporotic } \\
\text { medication }\end{array}$ & 8 & A & $0.40,7.15$ & $A$ & $\begin{array}{l}\text { 'Appropriate' added and an } \\
\text { expanded footnote to include calcium } \\
\text { and vitamin D }\end{array}$ \\
\hline 45 & $\begin{array}{l}\text { Patient using topical } \\
\text { corticosteroids does } \\
\text { NOT have itch or } \\
\text { discomfort that } \\
\text { interferes with daily } \\
\text { activities }\end{array}$ & $\mathrm{D}$ & $2.00,5.35$ & A & $\begin{array}{l}\text { Patient using topical } \\
\text { corticosteroids for } \\
\text { contact or allergic } \\
\text { dermatitis does not } \\
\text { have itch or discomfort } \\
\text { that interferes with daily } \\
\text { activities }\end{array}$ & - & & - & - & $\begin{array}{l}\text { This indicator was deleted by the } \\
\text { panel because there was no } \\
\text { identification of the diagnosis/ } \\
\text { condition being treated. However, } \\
\text { contact and allergic dermatitis is one } \\
\text { of the top } 40 \text { most frequently } \\
\text { managed problems by general } \\
\text { practitioners in patients } \geq 65 \text { years old } \\
\text { in Australia, }{ }^{36} \text { so this indicator was } \\
\text { re-worded by the authors }\end{array}$ \\
\hline
\end{tabular}


Table 2 Continued

\begin{tabular}{|c|c|c|c|c|c|c|c|c|c|}
\hline \multirow{2}{*}{$\begin{array}{l}\text { Criteria } \\
\text { number }\end{array}$} & \multirow{2}{*}{ 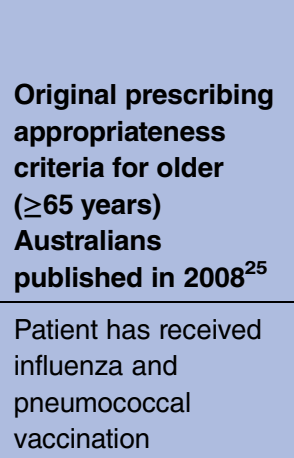 } & $\begin{array}{l}\text { Rating by } \\
\text { median } \\
\text { method }^{41} \\
\text { (median } \\
\text { value, } A \text {, } \\
\text { agreement; } D \text {, } \\
\text { disagreement), } \\
n=15\end{array}$ & \multicolumn{2}{|c|}{$\begin{array}{l}\text { Rating by IPRAS }{ }^{1} \\
\text { method }^{41} \text { (IPR value, } \\
\text { IPRAS value, A, } \\
\text { agreement; } \\
\text { D=disagreement), } n=15\end{array}$} & \multirow{2}{*}{$\begin{array}{l}\text { Validated prescribing } \\
\text { appropriateness } \\
\text { criteria for older } \\
\text { ( } \geq 65 \text { years) } \\
\text { Australians as a } \\
\text { result of this study } \\
\text { Patient has received } \\
\text { influenza and } \\
\text { pneumococcal } \\
\text { vaccination }\end{array}$} & $\begin{array}{l}\text { Rating by } \\
\text { median } \\
\text { method }^{41} \\
\text { (median value, } \\
\text { A, agreement, } D \text {, } \\
\text { disagreement), } \\
n=12\end{array}$ & \multicolumn{2}{|c|}{$\begin{array}{l}\text { Rating by IPRAS }{ }^{1} \\
\text { method }^{41} \text { (IPR value, } \\
\text { IPRAS value, A, } \\
\text { agreement, } D, \\
\text { disagreement), } n=12\end{array}$} & \multirow{2}{*}{$\begin{array}{l}\text { Amendment/reason } \\
\text { No change }\end{array}$} \\
\hline & & $A$ & $1.00,7.60$ & $A$ & & 9 & $0.00,8.35$ & A & \\
\hline 47 & $\begin{array}{l}\text { Patient has no } \\
\text { significant medication } \\
\text { interactions } \\
\text { (agreement between } \\
\text { two medication } \\
\text { interaction databases) }\end{array}$ & $D$ & $3.00,6.10$ & $A$ & $\begin{array}{l}\text { Patient has no clinically } \\
\text { significant medication } \\
\text { interactions (agreement } \\
\text { between two } \\
\text { medication interaction } \\
\text { databases) }\end{array}$ & 8 & $0.40,7.15$ & A & $\begin{array}{l}\text { 'Clinically' added to improve the } \\
\text { accuracy of the indicator }\end{array}$ \\
\hline 48 & $\begin{array}{l}\text { Patient has had no } \\
\text { significant change in } \\
\text { medications in the } \\
\text { previous } 90 \text { days }\end{array}$ & D & $1.20,3.25$ & A & Deleted & - & & - & $\begin{array}{l}\text { It was preferred to transfer this } \\
\text { information to the explanatory text of } \\
\text { the article }\end{array}$ \\
\hline New & & & & & $\begin{array}{l}\text { Patient taking thyroid } \\
\text { hormone replacement } \\
\text { therapy has had a } \\
\text { serum TSH } \\
\text { measurement within } \\
\text { the previous } 12 \text { months }\end{array}$ & & & & $\begin{array}{l}\text { Thyroid disease is a common } \\
\text { medical condition managed by GPs } \\
\text { in older Australians } 3669\end{array}$ \\
\hline New & & & & & $\begin{array}{l}\text { Patient with coronary } \\
\text { heart disease is taking } \\
\text { an } A C E I \text { or } A 2 A\end{array}$ & & & & $\begin{array}{l}\text { ACEls or A2As reduce the risk of } \\
\text { cardiovascular events }{ }^{70} 71 \text {. However, } \\
\text { a high incidence of comorbid disease } \\
\text { in CHD (commonly arthritis or } \\
\text { respiratory disease) or other clinical } \\
\text { factors (eg, dizziness or falls, } \\
\text { cognitive impairment, use of }>5 \\
\text { medicines, patient preference) may } \\
\text { be more important in determining } \\
\text { medication priorities }\end{array}$ \\
\hline
\end{tabular}


Table 3 Validated prescribing appropriateness criteria for older Australians ( $\geq 65$ years) for commonly used medications and medical conditions* $, \dagger, \ddagger\left({ }^{*}\right.$ for usage information for certain criteria, see table 4$)$

\begin{tabular}{|c|c|}
\hline $\begin{array}{l}\text { Criteria } \\
\text { number }\end{array}$ & Validated criteria \\
\hline 1 & Patient taking an antihypertensive is at the target blood pressure appropriate for them* \\
\hline 2 & Patient at high risk of a recurrent cardiovascular event is taking a statin* \\
\hline 3 & Patient with $\mathrm{CHD}$ or a history of $\mathrm{Ml}$ is taking a $\beta$-blocker \\
\hline 4 & Patient with $\mathrm{CHD}$ or a history of $\mathrm{MI}$ is taking an antiplatelet agent unless taking an oral anticoagulant \\
\hline 5 & Patient with $\mathrm{CHD}$ is taking an $\mathrm{ACEI}$ or $\mathrm{A} 2 \mathrm{~A}^{*}$ \\
\hline 6 & Patient with stable heart failure with HF-LVSD is taking a $\beta$-blocker \\
\hline 7 & Patient with stable heart failure with HF-LVSD is taking an ACEI or A2A* \\
\hline 8 & Patient with HF-LVSD or HFPEF is NOT taking medications which may exacerbate heart failure* \\
\hline 9 & $\begin{array}{l}\text { Patient with AF is taking an oral anticoagulant or an antiplatelet agent, depending on stroke risk and bleeding } \\
\text { risk }^{*}\end{array}$ \\
\hline 10 & Patient taking warfarin for AF has an INR between 2 and 3 \\
\hline 11 & $\begin{array}{l}\text { Patient with a history of non-haemorrhagic stroke or TIA is taking an antiplatelet agent unless taking an } \\
\text { anticoagulant }\end{array}$ \\
\hline 12 & Patient with risk factors for statin-induced myopathy is not taking a high dose of a high-potency statin* \\
\hline 13 & Patient with cardiovascular disease is NOT taking an NSAID \\
\hline 14 & $\begin{array}{l}\text { Patient with cardiovascular, respiratory disease or diabetes who smokes has been offered smoking cessation } \\
\text { options }{ }^{*}\end{array}$ \\
\hline 15 & Patient with type 2 diabetes and hypertension and albuminuria is taking an ACEI or A2A \\
\hline 16 & $\begin{array}{l}\text { Patient with diabetes at high risk of a cardiovascular event is taking an antiplatelet agent unless on an } \\
\text { anticoagulant }\end{array}$ \\
\hline 17 & $\begin{array}{l}\text { Patient with diabetes taking medications that may affect glycemic control is receiving regular monitoring of blood } \\
\text { glucose concentrations* }\end{array}$ \\
\hline 18 & Patient with diabetes has had an $\mathrm{HbA1c}$ measurement within the previous 6 months* \\
\hline 19 & Patient taking metformin for diabetes has had the dose adjusted for renal function* \\
\hline 20 & $\begin{array}{l}\text { Patient taking thyroid hormone replacement therapy has had a serum TSH measurement within the previous } \\
12 \text { months }\end{array}$ \\
\hline 21 & Patient with OA pain interfering with daily activities has been trialled on regular paracetamol $2-4 \mathrm{~g} /$ day \\
\hline 22 & Patient taking analgesic(s) has had the dose(s) titrated in order to avoid pain that interferes with daily activities \\
\hline 23 & Patient taking a regular opioid is on prophylactic treatment for constipation \\
\hline 24 & Patient with risk factors for impaired renal function is NOT taking an NSAID* \\
\hline 25 & Patient is NOT concurrently taking an ACEI or A2A, diuretic and NSAID (excluding low-dose aspirin) \\
\hline 26 & Patient has NOT been taking benzodiazepines for $>4$ weeks* \\
\hline 27 & Patient with a history of falls is NOT taking psychotropic medications* \\
\hline 28 & Patient taking an SSRI is NOT concurrently taking other medications that may contribute to serotonin toxicity* \\
\hline 29 & Patient with dementia is NOT receiving anticholinergic medication* \\
\hline 30 & Patient is not taking medication with SIGNIFICANT anticholinergic activity* \\
\hline 31 & $\begin{array}{l}\text { Patient taking a PPI is NOT taking a medication that may cause dyspepsia unless prescribed for } \\
\text { gastroprotection* }\end{array}$ \\
\hline 32 & Patient with COPD is NOT taking benzodiazepines \\
\hline 33 & Patient with asthma using an inhaled LABA is also using an inhaled corticosteroid \\
\hline 34 & $\begin{array}{l}\text { Patient using salbutamol or terbutaline inhaler more than three times per week for reversible airways disease } \\
\text { has been prescribed an ICS (except for exercise-induced asthma) }\end{array}$ \\
\hline 35 & Patient with asthma is NOT taking a medication that may worsen asthma* \\
\hline 36 & Patient with a UTI is not receiving nitrofurantoin or hexamine for prophylaxis or acute treatment \\
\hline 37 & Patient with a non-specific URTI is NOT receiving antibiotics* \\
\hline 38 & Patient with osteoporosis is receiving appropriate antiosteoporotic medication* \\
\hline 39 & Patient has received influenza and pneumococcal vaccination \\
\hline 40 & $\begin{array}{l}\text { Patient using topical corticosteroids for contact or allergic dermatitis does not have itch or discomfort that } \\
\text { interferes with daily activities }\end{array}$ \\
\hline 41 & $\begin{array}{l}\text { Patient has no clinically significant medication interactions (agreement between two medication interaction } \\
\text { databases)* }\end{array}$ \\
\hline
\end{tabular}

*These criteria are intended to be used by appropriately trained and qualified health professionals, as a tool to assist in making medication management decisions as part of the medication review process.

tPrior to the start of any medication, the contraindications and precautions for that medication should be considered.

$\ddagger$ The intended result of using these criteria is the reasonable and appropriate medication management of individual patients, rather than the systematic application of these criteria to all patients irrespective of other considerations.

$\mathrm{A} 2 \mathrm{~A}$, angiotensin 2 receptor antagonist; $\mathrm{ACEI}, \mathrm{ACE}$ inhibitor; $\mathrm{AF}$, atrial fibrillation; $\mathrm{CHD}$, coronary heart disease; COPD, chronic obstructive pulmonary disease; HbA1c, glycosylated haemoglobin; HF-LVSD, heart failure with left ventricular systolic dysfunction; HFPEF, heart failure with preserved ejection fraction; ICS, inhaled corticosteroid; INR, international normalised ratio; LABA, long-acting $\beta$ agonist; MI, myocardial infarct; NSAID, non-steroidal anti-inflammatory drug; OA, osteoarthritis; PPI, proton pump inhibitor; SSRI, selective serotonin reuptake inhibitor; TIA, transient ischaemic attack; TSH, thyroid stimulating hormone; UTI, urinary tract infection; URTI, upper respiratory tract infection. 
Table 4 Criteria usage information

\begin{tabular}{ll}
\hline $\begin{array}{l}\text { Criteria } \\
\text { number }\end{array}$ & Description of issue \\
\hline 1 & $\begin{array}{l}\text { Blood pressure targets } \\
(\mathrm{mm} \mathrm{Hg})\end{array}$ \\
2 & $\begin{array}{l}\text { Patients at high risk of a } \\
\text { cardiovascular event }(>15 \% \\
\text { within the next } 5 \text { years })\end{array}$
\end{tabular}

\section{Details}

Proteinuria $>1 \mathrm{~g} /$ day (with or without diabetes) $<125 / 75$. CHD, diabetes, chronic kidney disease, proteinuria ( $>300 \mathrm{mg} /$ day), stroke or $\mathrm{TIA}<130 / 80$. Others $<140 / 90 .{ }^{39}$ Current blood pressure guidelines may not be appropriate for all older patients, such as the oldest old; in palliative care; and for those who are/become hypotensive and/or fall ${ }^{47} 49-5273$

Age >75 years; history of diabetes, moderate or severe chronic kidney disease (persistent proteinuria, GFR $<60 \mathrm{ml} / \mathrm{min}$, eGFR $<45 \mathrm{ml} / \mathrm{min} / 1.73 \mathrm{~m}^{2}$ ),

hypercholesterolaemia (familial, TC>7.5 mmol/l), SBP $\geq 180$ or $\mathrm{DBP} \geq 110 \mathrm{~mm} \mathrm{Hg}$, ISH (SBP $\geq 160$ and $\mathrm{DBP} \leq 70 \mathrm{~mm} \mathrm{Hg}), \mathrm{CHD}$, stroke, TIA, $\mathrm{PAD}$, heart failure, aortic disease, $\mathrm{LVH}$, family history of premature CVD. ${ }^{39} 74$ The benefits of statins and risks of adverse effects are uncertain towards the end of life ${ }^{75}$

$4 \quad$ Antiplatelet agents and oral anticoagulants Use of $\mathrm{ACEl}$ or $\mathrm{A} 2 \mathrm{~A}$ in $\mathrm{CHD}$

Antiplatelet agents: aspirin, clopidogrel, dipyridamole and ticlopidine. Oral anticoagulants: dabigatran, phenindione, rivaroxaban and warfarin A high incidence of comorbid disease in CHD (typically arthritis and/or respiratory disease) or other clinical factors (eg, dizziness or falls, cognitive impairment, use of $>5$ medicines, patient preference) may be considerations in determining medication prescribing priorities ${ }^{30} 3472$

$8 \quad$ Medications that may exacerbate heart failure

HF-LVSD: anti-arrhythmic medicines (except for heart failure-specific $\beta$-blockers and amiodarone), non-dihydropyridine calcium-channel blockers (eg, verapamil or diltiazem), clozapine, corticosteroids, NSAIDs (excluding low-dose aspirin), thiazolidinediones, TNF- $\alpha$ inhibitors, topical $\beta$-blockers (when added to systemic $\beta$-blockers), tricyclic antidepressants. ${ }^{49} 7677$ HFPEF: venodilators (eg, isosorbide dinitrate), potent arterial vasodilators (eg, hydrallazine), digoxin (unless AF), excessive use of diuretics. Note; verapamil and diltiazem may improve diastolic function in $\mathrm{HFPEF}^{60}$

Stroke risk and bleeding risk

Stroke risk can be calculated using $\mathrm{CHADS}_{2}$ or $\mathrm{CHA}_{2} \mathrm{DS}_{2}-\mathrm{VASc}^{78}$ Risk factors for coumarin-related bleeding complications: advanced age, uncontrolled hypertension, history of MI or IHD, cerebrovascular disease, anaemia or a history of bleeding, concomitant use of aspirin/polypharmacy ${ }^{79}$

Risk factors for statin myopathy; high dose of high-potency statins

Smoking cessation options

Medications that may affect glycaemic control Age $>70$ years, presence of disease states (diabetes, hypothyroidism, renal and hepatic disease), concurrent use of ciclosporin, fibrates, CYP3A4 inhibitors (eg, diltiazem, macrolides, protease inhibitors, verapamil (except for pravastatin and rosuvastatin), severe intercurrent illness (infection, trauma and metabolic disorder), dose $\geq 40 \mathrm{mg}$ daily. High dose of high-potency statins ; $\geq 40 \mathrm{mg}$ atorvastatin or simvastatin; $>10 \mathrm{mg}$ rosuvastatin ${ }^{39} 80$

Counselling (extended, brief, telephone), support services (professional, family, social, work), pharmacotherapy

Increase blood glucose: baclofen, clozapine, ciclosporin, glucocorticoids, haloperidol, olanzapine, paliperidone, phenytoin, protease inhibitors, quetiapine, risperidone, sirolimus, tacrolimus and tricyclic antidepressants. Decrease blood glucose: excessive alcohol, disopyramide, perhexiline, quinine, trimethoprim/sulphamethoxazole ${ }^{39}$

Six-monthly $\mathrm{HbA1c}$ measurements

Treatment intensification in response to less than optimally controlled $\mathrm{HbA1c}$ may be inappropriate in patients with limited life expectancy or in frail older patients $^{8182}$

Metformin dose

Based on creatinine clearance: $60-90 \mathrm{ml} / \mathrm{min}$, maximum $2 \mathrm{~g}$ daily; $30-60 \mathrm{ml} /$ $\mathrm{min}$, maximum $1 \mathrm{~g}$ daily; $<30 \mathrm{ml} / \mathrm{min}$ avoid use. ${ }^{39}$ Based on eGFR: review dose if eGFR $<45 \mathrm{ml} / \mathrm{min} / 1.73 \mathrm{~m}^{2}$; avoid if eGFR $<30 \mathrm{ml} / \mathrm{min} / 1.73 \mathrm{~m}^{2}{ }^{83}$

Risk factors for impaired renal function

Benzodiazepine use

Volume depletion, age $>60$ years, salt-restricted diet, concomitant use of ACEls, A2As, ciclosporin or aspirin, GFR $\leq 60 \mathrm{ml} / \mathrm{min}$, cirrhosis, heart failure ${ }^{84}$ Benzodiazepines increase the risk of oversedation, ataxia, confusion, falls, respiratory depression and short-term memory impairment, and are recommended for short-term use only ${ }^{39}$ antipsychotics. ${ }^{85}{ }^{86}$ Medications causing (postural) hypotension 
Table 4 Continued

\section{Criteria \\ number Description of issue \\ Details}

28

Medications that may contribute to serotonin syndrome

29 and $30 \quad$ Medications with significant anticholinergic activity

Medications that may cause dyspepsia

Medications that may worsen asthma Non-specific URTI

Appropriate antiosteoporotic medication (eg, cardiovascular medicines) or cognitive impairment (eg, opioids) may also increase the risk of falls ${ }^{49} 87$

Antidepressants: desvenlafaxine, duloxetine, St John's wort, MAOls (including moclobemide), SSRIs, TCAs, venlafaxine. Opioids: dextromethorphan, fentanyl, pethidine, tramadol. Others: selegiline, linezolid, lithium, tryptophan ${ }^{39}$ Amantadine, amitriptyline, atropine*, belladonna alkaloids*, benzhexol, benztropine, biperiden, brompheniramine*, chlorpheniramine, chlorpromazine, clomipramine, clozapine, cyclizine, cyclopentolate, cyproheptadine*, darifenacin, dexchlorpheniramine* ${ }^{*}$ dimenhydrinate ${ }^{*}$, diphenhydramine*, disopyramide, dothiepin, doxepin, glycopyrrolate, homatropine, hyoscine* (butylbromide or hydrobromide), imipramine, ipratropium (nebulised), mianserin, nortriptyline, olanzapine, orphenadrine, oxybutynin, pericyazine, pheniramine ${ }^{*}$, pimozide, pizotifen, prochlorperazine, promethazine*, propantheline, solifenacin, tiotropium, tolterodine, trimeprazine*, trimipramine, triprolidine ${ }^{*}$, tropicamide ( ${ }^{*}$ available over-the-counter in Australia) ${ }^{39}$

Drugs with anticholinergic effects, aspirin, benzodiazepines, bisphosphonates, calcium channel antagonists, oral corticosteroids, dopaminergic drugs, doxycycline, erythromycin, ferrous sulphate, nitrates, NSAIDs, potassium chloride (slow release) 38394988

Aspirin, $\beta$-blockers (including eye drops), carbamazepine, echinacea, NSAIDs, royal jelly 3989

Acute bronchitis, pharyngitis, tonsillitis, non-suppurative otitis media and sinusitis $^{38}$

RDI of calcium from dietary sources and/or supplements $=1300-1500 \mathrm{mg}$ daily. $\mathrm{RDI}$ for vitamin $\mathrm{D}$ from sunlight and/or dietary sources and/or supplements $=600 \mathrm{IU}$ daily. Antiosteoporotic medication=bisphosphonates, calcitriol, denosumab, HRT, raloxifene, strontium, teriparatide. ${ }^{39}$ Evidence for fracture risk reduction in women $\geq 75$ years is either absent or lacking in NVF for alendronate, risedronate and teriparatide, and in HF for alendronate, risedronate, zoledronic acid and teriparatide. There are no data available for denosumab in VF, NVF or HF. ${ }^{90}$ The optimal duration of bisphosphonate therapy is uncertain. Evidence supports the use of strontium for 5 years, raloxifene for 4 years and zoledronic acid and denosumab for 3 years.

Exposure to teriparatide should be limited to 18 months. ${ }^{91}$ Data are limited for non-ambulatory patients and those with significant comorbidities. ${ }^{92}$ It should be noted that bone strength is only one of many determinants of fracture risk ${ }^{93}$ Medication interactions that may interfere with the outcome of therapy
Clinically significant

medication interactions

A2A, angiotensin 2 receptor antagonist; $\mathrm{ACEI}, \mathrm{ACE}$ inhibitor; $\mathrm{AF}$, atrial fibrillation; $\mathrm{CHADS}_{2}$, cardiac failure, hypertension, age, diabetes, stroke (doubled); $\mathrm{CHA}_{2} \mathrm{DS}_{2}-\mathrm{VASc}$, cardiac failure or dysfunction, hypertension, age over 75 years (doubled), diabetes, stroke (doubled), vascular disease, age 65-74 years, sex category (female); CHD, coronary heart disease; CVD, cardiovascular disease; DBP, diastolic blood pressure; GFR, glomerular filtration rate; HF, hip fracture; HF-LVSD, heart failure with left ventricular systolic dysfunction; HFPEF, heart failure with preserved ejection fraction; HRT, hormone replacement therapy; IHD, ischaemic heart disease; ISH, isolated systolic hypertension; LVH, left ventricular hypertrophy; MAOI, monoamine oxidase inhibitor; MI, myocardial infarct; NSAID, non-steroidal anti-inflammatory drug; NVF, non-vertebral fracture; PAD, peripheral arterial disease; RDI, recommended daily intake; SBP, systolic blood pressure; SSRI, selective serotonin reuptake inhibitor; TC, total cholesterol; TCA, tricyclic antidepressant; TIA, transient ischaemic attack; TNF, tumour necrosis factor; URTI, upper respiratory tract infection; VF, vertebral fracture.

diabetes and cardiovascular disease ${ }^{96}{ }^{97}$-diseaseoriented and drug-orientated criteria such as ours have shown good content, face, concurrent and predictive validity and operational feasibility, as well as use for internal and external quality assessment in both ambulatory and hospital care. ${ }^{35}$ Evidence-practice gaps in Australia have been identified in other areas besides diabetes and cardiovascular disease, such as in asthma, pain and vaccination status. ${ }^{98-101}$ The existence of these gaps formed part of the developmental process for these criteria.

\section{Prescribing appropriateness tools in Australia}

Appropriateness of prescribing has been assessed by measures that are explicit or implicit, in an effort to identify and reduce DRPs. ${ }^{102}$ In Australia, both types of measures have been used. ${ }^{103-107}$ However, they have been imported into the Australian healthcare environment, with consequent shortcomings related to both the intrinsic nature of the measure, as well as environment compatibility issues. For example, in a study evaluating the impact of Home Medicine Reviews on 
appropriateness of prescribing, a significant number of recommendations made regarding the need for monitoring and addition of missing therapy were found to have no impact on explicitly derived scores using the Medication Appropriateness Index, ${ }^{103}$ due to the intrinsic shortcomings of this tool. This is not a tool that gives precise guidance in relation to specific medicines. ${ }^{13}$

The Beers criteria, ${ }^{108}$ perhaps the tool most widely used to assess inappropriate prescribing in older people, has been used in Australia, but requires modification to exclude medicines not listed for government subsidy. ${ }^{107}$ This is because medicine availability and use in Australia is largely determined by the Australian Pharmaceutical Benefits Scheme ${ }^{37}$. Other Australian studies have found that some medicines listed as inappropriate by Beers may be appropriate for certain older people according to Australian practice; ${ }^{105}$ many medicines listed by Beers are not available in Australia; and that some medicines considered inappropriate in Australia are not listed by Beers. ${ }^{106}$ Disagreement between Beers and other criteria, such as the improving prescribing in the elderly tool, have been identified. ${ }^{109}$

The Beers criteria was recently updated, ${ }^{22}$ with approximately half the medicines listed being unavailable in Australia. Further, almost three quarters of the diseases or syndromes listed are not among the 40 problems most frequently managed in patients over 65 years of age by Australian general practitioners. ${ }^{97}$ Beers still contains recommendations to avoid some medicines that are recommended for certain older people in Australia such as amiodarone, and it has recently been shown that rhythm control in older patients with atrial fibrillation may be more effective than rate control in reducing mortality over the long term. ${ }^{110}$ Reviews of explicit and implicit criteria have identified these and other problems such as failure to address drug-drug interactions and drug duplication, errors in recommendations, underrepresentation of certain drug categories, inclusion of infrequently prescribed drugs, criteria that are inapplicable for all situations, disagreement between criteria and lack of organisation of criteria. ${ }^{45} 102111$

This has resulted in the development by others of criteria more suited to their own particular healthcare environment. ${ }^{112} 113$ Nationally based criteria have been described as the most desirable type of criteria, as they do not necessitate adaptation to local guidelines or national formularies before they can be used with confidence. $^{32}$ In 2008, we therefore sought to construct and validate a set of prescribing appropriateness criteria relevant to the Australian healthcare environment. Our development process differed from most other tools ${ }^{21} 1081^{112-117}$ as it did not initially involve a consensus panel, which has now been addressed. This development process also resulted in criteria unavailable in other tools such as monitoring, underprescribing, need for additional tests, evaluation of smoking and vaccination status, and certain drug interactions. ${ }^{32} 45 \quad 102$ Because we have generally named drug classes rather than specific drugs (table 3), and targeted common medical conditions found in older patients, ${ }^{118}{ }^{119}$ we anticipate that our work may have some international usefulness.

Despite a desire in Australia to develop decision support tools to improve healthcare quality, ${ }^{120}$ progress has consisted of the development of a limited number of non-age specific structure and process indicator lists for use in hospitals and general practice. ${ }^{40}{ }^{121-123}$ Many of these lists require updating. ${ }^{32} 113124$ Currently, there is no Australian prescribing appropriateness criteria list to assist in improving medication management in older people. The usefulness of such an approach has been acknowledged, together with other approaches such as medication review. ${ }^{125}$

\section{Co-morbidity}

Over $80 \%$ of older Australians have three or more chronic conditions, ${ }^{96}$ with Australian general practitioners shown to be dealing more frequently with patients presenting with three or four problems in the year 20092010 compared with $2000-2001 .{ }^{126}$ Comorbidity is associated with poor quality of life, physical disability, high healthcare use, multiple medicines with consequent increased risk of adverse drug events, fragmentation of care and increased mortality. ${ }^{119} 127$ Yet most Australian guidelines for chronic diseases do not modify or discuss the applicability of their recommendations to older patients with multiple comorbid conditions. ${ }^{34}$ This situation is not restricted to Australia. ${ }^{127}{ }^{128}$ Because the risk of harm in older patients increases in proportion to the number of treatments prescribed, prioritisation of therapeutic goals is necessary. For example, coronary heart disease (CHD) is an important morbidity in Australia ${ }^{77} 96$ for which treatment with ACE inhibitors or angiotensin 2 antagonists has been recommended to reduce the risk of cardiovascular events. ${ }^{70}{ }^{71}$ Other criteria derived outside Australia such as STOPP/START do not include this recommendation. ${ }^{21}$ However, the presence of comorbidity in CHD (commonly arthritis or respiratory disease) or other clinical factors (such as dizziness, falls or patient preference) may mean that medicines such as these are never started, due to consideration of other factors. While we wished to identify problems such as these, the ultimate decision regarding medicine use should always be made on a case-by-case basis based on clinical experience, a discussion between the healthcare professional and the patient, and best available evidence. ${ }^{72}$ Issues such as these may run counter to recommendations of disease-specific, evidence-based guidelines. ${ }^{34}$ Addition of our criteria with this associated usage information (table 4) to the implicit processes of Australian medication review may assist in addressing the problem of comorbidity.

\section{The oldest old}

Knowledge about the state of health and function of the oldest old is limited, ${ }^{129}$ with research on their drug use 
being scarce, and often based on small and selected samples without comparison with other age groups. ${ }^{130} 131$ We know that older patients in general are underrepresented in clinical trials, so that disease-specific guideline recommendations based on evidence may not apply to older cohorts. ${ }^{34}$ For example, undertreatment with antiosteoporotic medicines has been identified as a significant evidence-practice gap in Australia. ${ }^{98}$ While STOPP/START criteria recommend calcium and vitamin D supplements, ${ }^{21}$ no recommendations for more specific medicines are made. Further, evidence available for fracture risk reduction has been reported to differ with age. ${ }^{90}$ Similarly, blood pressure targets appropriate for older patients may not be appropriate for the oldest old, ${ }^{50}$ with adverse effects for antihypertensives found to be among the most frequent in centenarians. ${ }^{132}$ Issues regarding the oldest old appear in table 4, criteria 1, 2, 9, 18 and 39. We have attempted to achieve the advantages of using mostly explicit criteria, such as ease of application, with the addition of application information (tables 2 and 4) unavailable in our previous criteria set.

\section{Rationale for the use of the RAND/UCLA appropriateness method}

The RAND/UCLA appropriateness method has been used to rate lists ranging up to over 3000 indications, where panellists have been asked to use the clinical literature and their best clinical judgement to assess the appropriateness of performing a procedure. To do this, they have rated various clinical scenarios. ${ }^{46}$ While the number and type of our criteria may differ to this, similar criteria have been developed using the RAND/ UCLA method. For example, in the development of indicators for patients undergoing total hip or total knee replacement, 1 of the 68 indicators stated that for such patients, 'deep venous thrombosis prophylaxis should be provided for a minimum of 2 weeks after hospital discharge'. ${ }^{43}$ In the development of indicators for hazardous prescribing for general physicians (GPs) using this method, 1 of the 34 indicators identified the hazardous use of 'NSAID in a patient with heart failure'. ${ }^{44}$ We therefore followed a similar protocol.

\section{Nature of decision support tools}

Panel members emphasised that criteria may not provide definitive answers, instead indicating potential problems that might need addressing, due to a perceived unacceptable variation in care. ${ }^{133}$ While performance indicators are designed to measure the result of statements made in clinical practice guidelines, these guidelines often provide recommendations for care independent of other considerations such as multiple comorbidities, advanced age, frailty, patient preferences, disease burden or limited life expectancy. ${ }^{134-136}$ In such cases, less stringent goals, deprescribing or non-prescription may be more appropriate. $^{15} 81137$ For example, a frail older patient with multiple comorbidities and one or more functional impairments may have a life expectancy of approximately 2 years or less. ${ }^{75}$ This raises the question of whether failure to intensify treatment ${ }^{81}$ or to underuse evidencebased therapies ${ }^{138}$ reflects appropriate clinical judgement or an inappropriate care gap. The panel felt strongly that use of indicators, guidelines or criteria providing clinical decision support should never replace critical thinking in patient care. ${ }^{139}$

\section{Strengths and weaknesses}

We have followed a recommended approach ${ }^{120}$ by suggesting criteria for which high-quality evidence exists linking best practice with improved outcomes; where there are established evidence-practice gaps ${ }^{98}$ 99; and where the health conditions impose the greatest burden on the healthcare system. We used a validated consensus method, an expert panel of varied specialisation, and criteria written with the aim of conciseness and clarity.

In addition to face and content validity, these validated criteria, much like performance indicators, will require further developmental work to provide evidence of their acceptability, operational feasibility, reliability and degree of predictive validity. ${ }^{35133}$ Some of this work has already started with the original criteria. ${ }^{95}$ Further, these criteria only cover commonly occurring medicines and medical conditions. In addition, judgements made by an expert panel may not be representative of all healthcare professionals.

\section{Intended use}

These validated criteria are intended for use by healthcare providers to enhance the quality of the Australian medication review process, for quality improvement, educational purposes and internal audit. They are also intended for external quality assessment, such as use by policy makers and for public reporting. Stakeholder involvement will be critical to facilitate local uptake and encourage further research into the effects on health outcomes. $^{125}$

\section{CONCLUSION}

This study validated 41 prescribing appropriateness criteria to assist in identifying DRPs in older ( $\geq 65$ years) Australians. These criteria are intended to represent an addition to the medication management skill set that includes consideration of limited life expectancy, evidence base in the oldest old, drug burden and care coordination, patient and care-giver education, empowerment for self management, and shared decision-making. These skills are far from a 'do everything for everyone' philosophy, where aggressive treatment may encourage more care, not more appropriate care. ${ }^{31} 135$ Despite the presence of clinical decision support tools, healthcare 
providers need to know how to think about clinical problems, not just what to think. ${ }^{139}$

Contributors BJB designed and organised the study, analysed and interpreted the data and drafted the manuscript. TFC and RJM made substantial contributions to the conception, design, analysis and interpretation of the data and to critically revising the draft. All authors take responsibility for the accuracy and integrity of the study. All authors have given final approval of the version to be published.

Competing interests None.

Ethics approval Human Research Ethics Committee of the University of Sydney.

Provenance and peer review Not commissioned; externally peer reviewed.

Data sharing statement No additional data are available.

\section{REFERENCES}

1. Kwint $\mathrm{H}-\mathrm{F}$, Faber A, Gussekloo J, et al. Effects of medication review on drug-related problems in patients using automated drug-dispensing systems. Drugs Aging 2011;28:305-14.

2. Ellitt GR, Engblom E, Aslani $P$, et al. Drug related problems after discharge from an Australian teaching hospital. Pharm World Sci 2010;32:622-30

3. Nishtala PS, McLachlan AJ, Bell JS, et al. A retrospective study of drug-related problems in Australian aged care homes: medication reviews involving pharmacists and general practitioners J Eval Clin Pract 2011;17:97-103.

4. Stafford AC, Tenni PC, Peterson GM, et al. Drug-related problems identified in medication reviews by Australian pharmacists. Pharm World Sci 2009;31:216-23.

5. Hilmer SN, Gnjidic D. The effects of polypharmacy in older adults. Clin Pharmacol Ther 2009;85:86-8.

6. Pirmohamed M, James S, Meakin S, et al. Adverse drug reactions as cause of admission to hospital: prospective analysis of 18820 patients. BMJ 2004;329:15-19.

7. Kongkaew C, Noyce PR, Ashcroft DM. Hospital admissions associated with adverse drug reactions: a systematic review of prospective observational studies. Ann Pharmacother 2008;42:1017-25.

8. Castelino RL, Chen TF, Guddattu V, et al. Use of evidence-based therapy for the prevention of cardiovascular events among older people. Eval Health Prof 2010;33:276-301.

9. Heeley EL, Peiris DP, Patel AA, et al. Cardiovascular risk perception and evidence-practice gaps in Australian general practice (the AusHEART study). MJA 2010;192:254-9.

10. National Institute of Clinical Studies, Australian Government. Evidence-practice gaps report, vol. 1. A review of developments 2004-2007. 2008. http://www.nhmrc.gov.au/nics/materials-andresources/evidence-practice-gaps-report-volume-one-reviewdevelopments-2004-2007 (accessed 18 Jul 2012).

11. Gilbert AL, Roughead EE, Beilby J, et al. Collaborative medication management services: improving patient care. MJA 2002:177:189-92.

12. Elliott RA, Martinac G, Campbell S, et al. Pharmacist-led medication review to identify medication-related problems in older people referred to an aged care assessment team. A randomiized comparative study. Drugs Aging 2012;29:593-605.

13. Kaur S, Mitchell G, Vitetta L, et al. Interventions that can reduce inappropriate prescribing in the elderly. Drugs Aging 2009;26:1013-28.

14. Tulner LR, van Campen JPCM, Frankfort SV, et al. Changes in under-treatment after comprehensive geriatric assessment. An observational study. Drugs Aging 2010;27:831-43.

15. Le Couteur DG, Banks E, Gnjidic D, et al. Deprescribing. Aust Presc 2011;34:182-5.

16. Garfinkel D, Mangin D. Feasibility study of a systemic approach for discontinuation of multiple medications in older adults. Arch Intern Med 2010;170:1648-54.

17. Gill JM, Mainous AG, Koopman RJ, et al. Impact of EHR-based clinical decision support on adherence to guidelines for patients on NSAID's: a randomised controlled trial. Ann Fam Med 2011;9:22-30.

18. O'Connor PJ, Sperl-Hillen JM, Rush WA, et al. Impact of elecronic health record clinical decision support on diabetes care; a randomised trial. Ann Fam Med 2011;9:12-21.
19. Chang C-B, Chan D-C. Comparison of published explicit criteria for potentially inappropriate medications in older adults. Drugs Aging 2010;27:947-57.

20. Scott I, Jayathissa S. Quality of drug prescribing in older patients: is there a problem and can we improve it? Intern Med J 2010:40:7-18.

21. Gallagher P, Ryan C, Byrne S, et al. STOPP (screening tool of older person's prescriptions) and START (screening tool to alert doctors to right treatment). Consensus validation. Int J Clin Pharmacol Ther 2008;46:72-83.

22. American Geriatrics Society updated Beers criteria for potentially inappropriate medication use in older adults. The American Geriatrics Society 2012 Beers Criteria update expert panel. J Am Geriatr Soc 2012;60:616-31.

23. Medicare Australia [homepage on the internet]. Australian Government. Home Medicines Review. http://www. medicareaustralia.gov.au/provider/pbs/fourth-agreement/hmr.jsp (accessed 17 Jul 2012).

24. Pharmaceutical Society of Australia. Guidelines for pharmacists providing Residential Medication Management Review (RMMR) and Quality Use of Medicines (QUM) services. 2011. http://www. psa.org.au/download/practice-guidelines/rmmr-and-qum-services. pdf (accessed 17 Aug 2012).

25. Basger BJ, Chen TF, Moles RJ. Inappropriate medication use and prescribing indicators in elderly Australians. Drugs Aging 2008;25:777-93

26. Chang C-B, Chen J-H, Wen C-J, et al. Potentially inappropriate medications in geriatric outpatients with polypharmacy: application of six sets of published explicit criteria. Br J Clin Pharmacol 2011;72:482-89.

27. Laroche ML, Charmes JP, Bouthier JP, et al. Inappropriate medications in the elderly. Clin Pharmacol Ther 2009;85:94-7.

28. Maio V, Jutkowitz E, Herrera $\mathrm{K}$, et al. Appropriate medication prescribing in elderly patients: how knowledgeable are primary care physicians? A survey study in Parma, Italy. J Clin Pharm Ther 2011;36:468-80.

29. Resnick B, Pacala JT. 2012 Beers criteria. J Am Geriatr Soc 2012;60:612-13

30. Fried TR, Tinetti ME, lannone MA. Primary care clinicians experiences with treatment decision making for older persons with multiple conditions. Arch Intern Med 2011;171:75-80.

31. Hayward RA. Performance measurement in search of a path. N Eng J Med 2007;356:951-2.

32. Dimitrow MS, Airaksinen MSA, Kivela S-L, et al. Comparison of prescribing criteria to evaluate the appropriateness of drug treatment in individuals aged 65 and older: a systematic review. J Am Geriatr Soc 2011:59:1521-30.

33. Mangin D, Sweeney K, Heath I. Preventative health care in elderly people needs rethinking. BMJ 2007;335:285-7.

34. Scott IA, Guyatt GH. Cautionary tales in the interpretation of clinical studies involving older persons. Arch Intern Med 2010;170:587-95.

35. Martirosyan L, Voorham J, Haaijer-Ruskamp FM, et al. A systematic literature review: prescribing indicators related to type 2 diabetes mellitus and cardiovascular risk management. Pharmacoepidemiol Drug Saf 2010;19:319-34.

36. Bettering the evaluation and care of health $(\mathrm{BEACH})$. Family Medicine Research Centre. The University of Sydney. http://sydney. edu.au/medicine/fmrc/beach/ (accessed 18 Jul 2012).

37. Access to Pharmaceutical Services. Pharmaceutical Benefits Scheme. Department of Health and Ageing. Australian Government. http://www.health.gov.au/internet/budget/publishing. nsf/Content/2010-11_Health_PBS_sup1/\$File/Outcome $\% 202 \%$ 20-\%20Access\%20to\%20Pharmaceutical\%20Services.pdf (accessed $10 \mathrm{Jul}$ 2012).

38. eTG complete [CD-ROM]. Melbourne (VIC): Therapeutic Guidelines: 2011 Nov.

39. Rossi S, ed. Australian Medicines Handbook 2012 (CD-ROM) Adelaide: Australian Medicines Handbook Pty Ltd, 2012.

40. NSW Therapeutic Advisory Group. Indicators for Quality Use of Medicines in Australian Hospitals 2007 http://www.ciap.health.nsw. gov.au/nswtag/documents/publications/QUMIndicators/ Manual0408.pdf (accessed 18 Jul 2012).

41. Fitch K, Bernstein SJ, Aguilar MS, et al. The RAND/UCLA Appropriateness Method User's Manual 2001. Rand Corporation. http://www.rand.org/pubs/monograph_reports/MR1269.html (accessed 18 Jul 2012).

42. McGory ML, Kao KK, Shekelle PG, et al. Developing quality indicators for elderly surgical patients. Ann Surg 2009;250:338-47.

43. SooHoo NF, Lieberman JR, Farng E, et al. Development of quality of care indicators for patients undergoing total hip or total knee replacement. Qual Saf 2011;20:153-7. 
44. Avery AJ, Dex GM, Mulvaney C, et al. Development of prescribing-safety indicators got GPs using the RAND appropriateness method. Brit J Gen Pract 2011;61:526-36.

45. Levy HB, Marcus E-L, Christen C. Beyond the Beers criteria: a comparative overview of explicit criteria. Ann Pharmacother 2010;44:1968-75

46. Shekelle PG. Appropriateness criteria: a useful tool for the cardiologist. Heart 2009;95:517-20.

47. Hypertension and target blood pressure. National Prescribing Service. Indicators of quality prescribing in Australian general practice. A Manual for Users [online]. http://www.nps.org.au/_data/assets/ pdf file/0019/37351/indicators full.pdf (accessed $18 \mathrm{Jul} 2012$ ).

48. Heart Foundation Guide to management of hypertension 2008 Updated December 2010. http://www.heartfoundation.org.au/ SiteCollectionDocuments/HypertensionGuidelines2008to 2010Update.pdf (accessed 18 Jul 2012).

49. Rossi S, ed. Australian medicines handbook drug choice companion: aged care. 3rd edn. Adelaide: Australian Medicines Handbook Pty Ltd, 2010.

50. Charpentier MM, Bundeff MM. Treating hypertension in the very elderly. Ann Pharmacother 2011;45:1138-45.

51. Gribbin J, Hubbard R, Gladman JRF, et al. Risk of falls associated with antihypertensive medication; population-based case control study. Age Ageing 2010;39:592-7.

52. Tinetti ME, Kumar $\mathrm{C}$. The patient who falls. JAMA 2010;303:258-66.

53. Therapeutics Initiative. Do statins have a role in primary prevention? An update. Ther Lett 2010;77:1-5. http://www.ti.ubc.ca/ sites/ti.ubc.ca/files/77.pdf (accessed 18 Jul 2012).

54. Ray KK, Seshasai SRK, Erqou S, et al. Statins and all-cause mortality in high-risk primary prevention. A meta-analysis of 11 randomised controlled trials involving 65,229 participants. Arch Intern Med 2010;170:1024-31.

55. deLorgeril M, Salen P, Abramson J, et al. Cholesterol lowering, cardiovascular diseases, and the rosuvastatin-JUPITER controversy. A critical reappraisal. Arch Intern Med 2010;170:1032-6.

56. Petersen LK, Christensen K, Kragstrup J. Lipid-lowering treatment to the end? A review of observational studies and RCTs on cholesterol and mortality in 80+-year olds. Age Ageing 2010;39:674-80.

57. Robinson JG. Lipid-lowering therapy for the primary prevention of cardiovascular disease in the elderly. Opportunities and challenges. Drugs Aging 2009;26:917-31.

58. Krum H, Jelinek M, Stewart S, et al. 2011 Update to National Heart Foundation of Australia and Cardiac Society of Australia and New Zealand guidelines for the prevention, detection and management of chronic heart failure in Australia 2006. MJA 2011;194:405-9.

59. Krum H, Teerlink JR. Medical therapy for chronic heart failure. Lancet 2011;378:713-21.

60. Huang D, Cheng JWM. Pharmacologic management of heart failure with preserved ejection fraction. Ann Pharmacother 2010;44:1933-45.

61. Management of diastolic heart failure [revised 2008 June]. In: eTG complete [CD-ROM]. Melbourne: Therapeutic Guidelines Limited, 2011.

62. Huang N, Duggan K, Harman J. Lifestyle management of hypertension. Aust Presc 2008;31:150-3.

63. Stokes GS. Management of hypertension in the elderly patient. Clin Interv Aging 2009;4:379-89.

64. Bajorek BV, Krass I, Ogle SJ, et al. Optimizing the use of antithrombotic therapy for atrial fibrillation in older people: a pharmacist-led multidisciplinary intervention. J Am Geriatr Soc 2005;53:1912-20.

65. De Caterina R, Hylek EM. Stroke prevention in atrial fibrillation: current status and near-future directions. Am J Med 2011;124:793-9

66. Gillett RG, Norrell A. Considerations for safe use of statins: liver enzyme abnormalities and muscle toxicity. Am Fam Physician 2011;83:711-16

67. Thomas JE, Tershakovec AM, Jones-Burton C, et al. Lipid lowering for secondary prevention of cardiovascular disease in older adults. Drugs Aging 2010;27:959-72.

68. Perrotta C, Aznar M, Mejia R, et al. Oestrogens for preventing recurrent urinary tract infection in postmenopausal women. Cochrane Database Syst Rev2008; (2): CD005131. doi:10.1002/ 14651858.CD005131.pub2

69. Treatment of hypothyroidism [revised 2009 June]. In: eTG complete [CD-ROM]. Melbourne: Therapeutic Guidelines Limited, 2011.

70. Reducing Risk in Heart Disease 2007. A summary guide for preventing cardiovascular events in people with coronary heart disease. Updated 2008. National Heart Foundation of Australia and the Cardiac Society of Australia and New Zealand. http:// www.heartfoundation.org.au/information-for-professionals/ Clinical-Information/Pages/coronary-heart-disease.aspx (accessed 12 Jul 2012)

71. Volpe M. Should all patients at high cardiovascular risk receive renin-angiotensin system blockers? Q J Med 2012;105:11-27.

72. Boyd CM, Leff B, Wolff JL, et al. Informing clinical practice guideline development and implementation: prevalence of coexisting conditions among adults with coronary heart disease. J Am Geriatr Soc 2011;59:797-805.

73. Heart Foundation. Guide to management of Hypertension 2008 updated 2010 [online].http://www.heartfoundation.org.au/ SiteCollectionDocuments/HypertensionGuidelines2008to2010 Update.pdf (accessed 18 Jul 2012).

74. Guidelines for the assessment of absolute cardiovascular disease risk. http://www.heartfoundation.org.au/SiteCollectionDocuments/ guidelines-Absolute-risk.pdf (accessed 18 Jul 2012).

75. Holmes HM. Rational prescribing for patients with a reduced life expectancy. Clin Pharmacol Ther 2009;85:103-7.

76. Improving treatment of systolic heart failure. NPS News 75, 2011 National Prescribing Service Limited. http://www.nps.org.au/_data/ assets/pdf_file/0006/132495/News75_heart_failure_1011v2.pdf (accessed 18 Jul 2012)

77. Caughey GE, Roughead EE, Shakib S, et al. Co-morbidity and potential treatment conflicts in elderly heart failure patients. Drugs Aging 2011;28:575-81.

78. Lip GYH, Halperin JL. Improving stroke risk stratification in atrial fibrillation. Am J Med 2010;123:484-8.

79. Hughes M, Lip GYH. Risk factors for anticoagulation-related bleeding complications in patients with atrial fibrillation: a systematic review. Q J Med 2007;100:599-607.

80. Adverse Drug Reactions Advisory Committee. Statins and muscle disorders-be careful with the dose. Aust Adverse Drug React Bull 2008;27. http://www.tga.gov.au/pdf/aadrb-0806.pdf (accessed 18 Jul 2012)

81. LeChauncy WD, Landrum CR, Urech TH, et al. Treating chronically ill people with diabetes mellitus with limited life expectancy: Implications for performance measurement. J Am Geriatr Soc 2012;60:193-201.

82. Lee SJ, Boscardin WJ, Cenzer IS, et al. The risks and benefits of implementing glycemic control guidelines in frail older adults with diabetes mellitus. J Am Geriatr Soc 2011;59:666-72.

83. Joint Formulary Committee. British National Formulary (online) London: BMJ Group and Pharmaceutical Press <http://www. medicinescomplete.com> (accessed 18 Jul 2012).

84. Analgesic choices in persistent pain. Prescribing Pract Rev 2006;35. National Prescribing Service Limited http://www.nps.org. au/ data/assets/pdf file/0017/23750/ppr35_analgesics_0906.pdf (accessed 18 Jul 2012)

85. Hill KD, Wee R. Psychotropic drug-induced falls in older people. A review of interventions aimed at reducing the problem. Drugs Aging 2012;29:15-30.

86. Zeimer H. Medications and falls in older people. J Pharm Pract Res 2008;38:148-51.

87. Caughey GE, Roughead EE, Shakib S, et al. Comorbidity of chronic disease and potential treatment conflicts in older people dispensed antidepressants. Age Ageing 2010;39:488-94.

88. Proton pump inhibitors. NPS News 46, 2006. National Prescribing Service Limited. http://www.nps.org.au/ data/assets/ pdf_file/0016/23821/news46_gord_ppis_0606.pdf (accessed 18 Jul 2012)

89. National Asthma Council Australia. Asthma Management Handbook 2006. Australia, Melbourne: National Asthma Council, 2006 http://www.nationalasthma.org.au/uploads/handbook/ 370-amh2006 web 5.pdf (accessed 18 Jul 2012).

90. Inderjeeth CA, Poland KE. Management of osteoporosis in older people. J Pharm Pract Res 2010;40:229-34.

91. Reducing fracture risk in osteoporosis. NPS News 73, 2011. National Prescribing Service Limited. http://www.nps.org.au/_data/ assets/pdf file/0014/126401/News73 osteoporosis_0511.pdf (accessed 18 Jul 2012).

92. Gates BJ, Sonnett TE, DuVall CAK, et al. Review of osteoporosis pharmacotherapy for geriatric patients. Am J Geriatr Pharmacother 2009;7:293-323

93. Cheung AM, Detsky AS. Osteoporosis and fractures-missing the bridge? JAMA 2008;299:1468-70.

94. Commonwealth of Australia. The National Strategy for Quality Use of Medicines Plain English Edition [online]. http://www.health.gov. au/internet/main/Publishing.nsf/Content/CA777524C860DFF2CA 256F1800468B61/\$File/natstrateng.pdf (accessed 18 Jul 2012). 
95. Basger BJ, Chen TF, Moles RJ. Application of a prescribing indicators tool to assist in identifying drug-related problems in a cohort of older Australians. Int J Pharm Pract 2012;20:172-82.

96. Caughey GE, Vitry Al, Gilbert AL, et al. Prevalence of comorbidity of chronic diseases in Australia. BMC Public Health 2008. http:// www.biomedcentral.com/1471-2458/8/221 (accessed 18 Jul 2012).

97. Top 40 most frequently managed problems: Patients aged 65+. Personal communication, unpublished data from the BEACH Program, Family Medicine Research Centre, University of Sydney, November 2011. http://sydney.edu.au/medicine/fmrc/beach/index. php (accessed 10 Jul 2012).

98. Evidence-practice gaps report. Vol. 2. National Institute of Clinical Studies. Australian Government 2005. http://www.nhmrc.gov.au/ nics/material resources/resources/evidence volume two.htm (accessed 12 Jul 2012)

99. Evidence-practice gaps report. Vol. 1. National Institute of Clinical Studies, Australian Government 2003. http://www.nhmrc.gov.au/ nics/material_resources/resources/evidence_volume_one.htm (accessed 17 July 2012).

100. Evidence-practice gaps report. Vol 1. A review of developments: 2004-2007. National Institute of Clinical Studies. Australian Government. http://www.nhmrc.gov.au/nics/materials-andresources/evidence-practice-gaps-report-volume-one-reviewdevelopments-2004-2007 (accessed 17 July 2012).

101. Bajorek BV, Ren S. Utilisation of antithrombotic therapy for stroke prevention in atrial fibrillation in a Sydney hospital: then and now. Int J Clin Pharm 2012;34:88-97.

102. O'Connor MN, Gallagher P, O'Mahony D. Inappropriate prescribing Criteria, detection and prevention. Drugs Aging 2012;29:437-52.

103. Castelino RL, Bajorek BV, Chen TF. Retrospective evaluation of home medicine review by pharmacists in older Australian Patients using the Medication Appropriateness Index. Ann Pharmacother 2010;44:1922-9.

104. King MA, Roberts MS. The influence of the Pharmaceutical Benefits Scheme (PBS) on inappropriate prescribing in Australian nursing homes. Phar World Sci 2007;29:39-42.

105. Stafford AC, Alswayan MS, Tenni PC. Inappropriate prescribing in older residents of Australian care homes. J Clin Pharm Ther 2011;36:33-44.

106. Widagdo IS, Nyfort-Hansen K, Kowalski SR. Prevalence of potentially inappropriate medication use in elderly hospitalised patients. J Pharm Pract Res 2011;41:122-5.

107. Roughead EE, Anderson B, Gilbert AL. Potentially inappropriate prescribing among Australian veterans and war widows/widowers. Intern Med J 2007;37:402-5.

108. Fick DM, Cooper JW, Wade WE, et al. Updating the Beers criteria for potentially inappropriate medication use in older adults: results of a US consensus panel of experts. Arch Intern Med 2003;163:2716-24.

109. Ryan C, O'Mahony D, Kennedy J, et al. Appropriate prescribing in the elderly: an investigation of two screening tools, Beers criteria considering diagnosis and independent of diagnosis and improving prescribing in the elderly tool to identify inappropriate use of medicines in the elderly in primary care in Ireland. $J$ Clin Pharm Ther 2009;34:369-76.

110. Ionescu-Ittu R, Abrahamowicz M, Jackevicius CA, et al Comparative effectiveness of rhythm control vs rate control drug treatment effect on mortality in patients with atrial fibrillation. Arch Intern Med 2012;172:997-1004.

111. Page RL, Linnebur SA, Bryant LL, et al. Inappropriate prescribing in the hospitalised elderly patient: defining the problem, evaluation tools, and possible solutions. Clin Interv Aging 2010;5:75-87.

112. Holt S, Schmiedl S, Thurmann PA. Potentially inappropriate medications in the elderly: the PRISCUS list. Dtsch Arzteblatt Int 2010;107:543-51.

113. Laroche ML, Charmes JP, Merle L. Potentially inappropriate medications in the elderly: a French consensus panel list. Eur $J$ Clin Pharmacol 2007;63:725-31.

114. Beers $\mathrm{MH}$. Explicit criteria for determining potentially inappropriate medication use by the elderly. An update. Arch Intern Med 1997; 157:1531-6.

115. Beers MH, Ouslander JG, Rollingher I, et al. Explicit criteria for determining inappropriate medication use in nursing home residents. UCLA Division of Geriatric MedicineArch Intern Med 1991;151:1825-32.

116. McLeod PJ, Huang AR, Tamblyn RM, et al. Defining inappropriate practices in prescribing for elderly people: a national consensus panel. CMAJ 1997:156:385-91.
117. Zhan C, Sangl J, Bierman AS, et al. Potentially inappropriate medication use in the community-dwelling elderly: findings from the 1996 Medical Expenditure Panel Survey. JAMA 2001:286:2823-9.

118. Starfield B, Lemke KW, Herbert R, et al. Comorbidity and the use of primar care and specialist care in the elderly. Ann Fam Med 2005;3:215-22.

119. Barnett K, Mercer SW, Norbury M, et al. Epidemiology of multimorbidity and implications for health care, research, and medical education: a cross-sectional study. Lancet 2012;380:37-43.

120. Evans SM, Lowinger JS, Sprivulis PC, et al. Prioritizing quality indicator development across the healthcare system: identifying what to measure. Intern Med J 2009;39:648-54.

121. Elliott RA, Woodward MC, Oborne CA. Indicators of prescribing quality for elderly hospital inpatients. Aust $J$ Hosp Pharm 2001;31:19-25.

122. National Prescribing Service. Indicators of quality prescribing in Australian general practice 2006. A Manual for Users. http://www. nps.org.au/_data/assets/pdf_file/0019/37351/indicators_full.pdf (accessed $18 \mathrm{Jul}$ 2012).

123. A proposed set of clinical indicators for stakeholder comment by 30 July 2012. RACGP clinical improvement unit practice, policy and innovation department. The Royal Australian College of General Practitioners. http://www.racgp.org.au/Content/NavigationMenu/ ClinicalResources/Clinicalindicators/2012Clinicallndicators_ ConsultationPaper.pdf (accessed 16 Jul 2012).

124. Morris CJ, Cantrill JA, Hepler CD, et al. Preventing drug-related morbidity - determining valid indicators. Int J Qual Health Care 2002;14:183-98

125. Bell JS, Le Couteur DG, McLachlan AJ, et al. Improving medicine selection for older people. Do we need an Australian classification for inappropriate medicines use? Aust Fam Physician 2012;41:9-10.

126. Britt $\mathrm{H}$, Miller GC, Charles J, et al. General practice activity in Australia 2000-1 to 2009-10: 10 year data tables. General practice series no. 28. Cat. no. GEP 28. Canberra: AlHW, 2010. http://www. aihw.gov.au/publication-detail/?id=6442472440\&tab=2 (accessed 17 Jul 2012)

127. Boyd CM, Darer J, Boult C, et al. Clinical practice guidelines and quality of care for older patients with multiple comorbid diseases. JAMA 2005;294:716-24.

128. Kamerow D. How can we treat multiple chronic conditions? BMJ 2012;344:e1487. doi:10.136/bmi.e87

129. Richmond RL, Law J, Kay-Lambkin F. Physical, mental, and cognitive function in a convenience sample of centenarians in Australia. J Am Geriatr Soc 2011:59:1080-6.

130. Wastesson JW, Parker MG, Fastbom J, et al. Drug use in centenarians compared with nonagenarians and octogenarians in Sweden: a nationwide register-based study. Age Ageing 2012;41:218-24

131. Watts G. Why the exclusion of older people from clinical research must stop. BMJ 2012;344:e3445.

132. Frada G, Bennati E, Cardillo E, et al. Pharmacotherapy in the extreme longevity. Arch Gerontol Geriatr 2009;49:60-3.

133. Campbell SM, Braspenning J, Hutchinson A, et al. Research methods used in developing and applying quality indicators in primary care. Qual Saf Health Care 2002;11:358-64.

134. Vitry Al, Zhang Y Quality of Australian clinical guidelines and relevance to the care of older people with multiple comorbid conditions. Med J Aust 2008;189:360-5.

135. Lee SJ, Walter LC. Quality indicators for older adults. Preventing unintended harms. JAMA 2011;306:1481-2.

136. Gilbert AL, Caughey GE, Vitry Al, et al. Ageing well: improving the management of patients with multiple chronic health problems. Australas J Ageing 2011;30(S2):32-7.

137. Gnjidic D, Hilmer SN, Blyth FM, et al. High-risk prescribing and incidence of frailty among older community-dwelling men. Clin Pharmacol Ther 2012;91:521-8.

138. Sirois C, Moisan J, Poirier $P$, et al. Association between age and the initiation of antihypertensive, lipid lowering and antiplatelet medications in elderly individuals newly treated with antidiabetic drugs. Age Ageing 2009;38:741-5.

139. Powers JH. Practice guidelines. Belief, criticism, and probability. Arch Intern Med 2011:171:15-16. 